\title{
Transmission Line Capital Costs
}
K. R. Hughes
D. R. Browńn

May 1995

Prepared for the U.S. Dêpartment of Energy under Contract DE-AC06-76RLO 1830

Pacific Northwest Laboratory Operated for the U.S. Department of Energy by Battelle-Memórial Institute

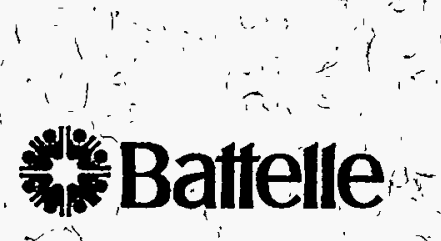


This report was prepared as an account of work sponsored by an âgency of the United States Government. Neither the United States Government nor any agency. 'thereof, nor Battelle, Mémorial Irstitute, nor any of their emplóyees, makes any warranty, expressed or implied, or assumes any legal liability or responsibility for. the accuracy, cómpleteness, orusefulness of any information, apparatus, product, or process discloséd, or represents that its use would not infringe privately owned rights. Reference herein to any specific commercial product; process, or service by trade name, trademark, manufacturer, or otherwise does not necessarily constitute ;or imply: its endorsement, recommendation, 'or favoring by the United States Government or any agency thereof, or Battelle Memorial Institute. The views and opinions of authors expressed herein do not necessarily state or reflect those of the United States Government or any agency thereof.

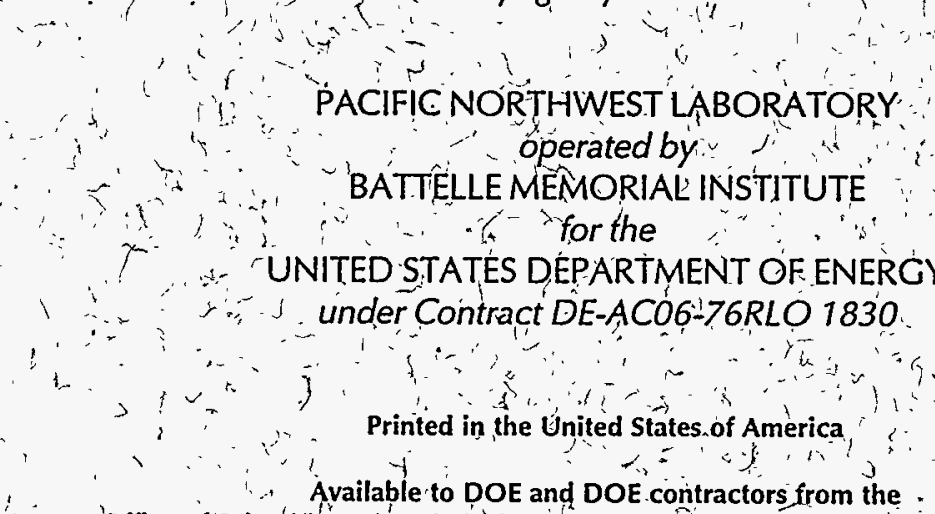

Office of Scientific and Technical Information, P.O. Box 62, Oak Ridge, TN 37831;

h. prices available from '(615) 576-8401. FTS 626-8401.

Available to the public from the National Technical Information Service, U.S. Department of Cómmerce, 5285 Port Royal Rd., Sprinğfield,'VA 22161,

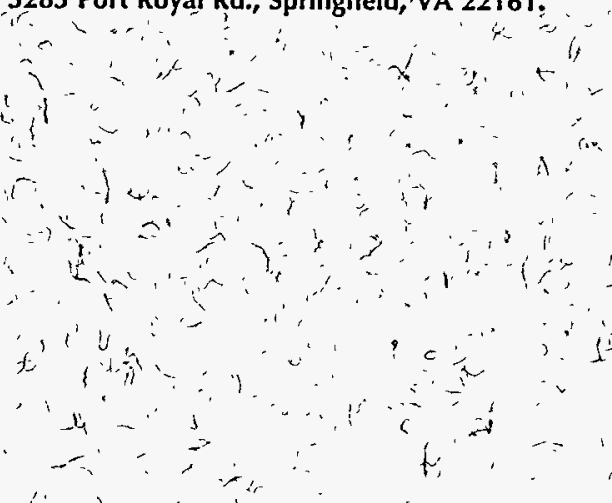




\section{DISCLAIMER}

Portions of this document may be illegible in electronic image products. Images are produced from the best available original document. 


\title{
Transmission Line Capital Costs
}

\author{
K. R. Hughes \\ D. R. Brown
}

May 1995

Prepared for the U.S. Department of Energy under Contract DE-AC06-76RLO 1830

Pacific Northwest Laboratory

Richland, Washington 99352 


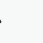




\section{Summary}

The displacement or deferral of conventional AC transmission line installation is a key benefit associated with several technologies being developed with the support of the U.S. Department of Energy's Office of Energy Management (OEM). Previous benefits assessments conducted within OEM have been based on significantly different assumptions for the average cost per mile of AC transmission line. In response to this uncertainty, an investigation of transmission line capital cost data was initiated. The objective of this study was to develop a database for preparing preliminary estimates of transmission line costs.

An extensive search of potential data sources identified databases maintained by the Bonneville Power Administration (BPA) and the Western Area Power Administration (WAPA) as superior sources of transmission line cost data. The BPA and WAPA data were adjusted to a common basis and combined together. The composite database covers voltage levels from 13.8 to $765 \mathrm{kV}$, with cost estimates for a given voltage level varying depending on conductor size, tower material type, tower frame type, and number of circuits. Reported transmission line costs vary significantly, even for a given voltage level. This can usually be explained by variation in the design factors noted above and variation in environmental and land (right-of-way) costs, which are extremely site-specific.

Cost estimates prepared from the composite database were compared to cost data collected by the Federal Energy Regulatory Commission (FERC) for investor-owned utilities from across the United States. The comparison was hampered because the only design specifications included with the FERC data were voltage level and line length. Working within this limitation, the FERC data were not found to differ significantly from the composite database. Therefore, the composite database was judged to be a reasonable proxy for estimating national average costs: 
. 


\section{Contents}

Summary $\quad \ldots \ldots \ldots \ldots \ldots \ldots \ldots \ldots \ldots \ldots \ldots \ldots \ldots \ldots \ldots \ldots \ldots \ldots \ldots \ldots$

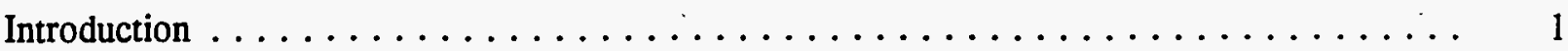

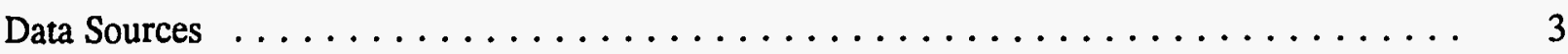

Composite Database $\ldots \ldots \ldots \ldots \ldots \ldots \ldots \ldots \ldots \ldots \ldots \ldots \ldots \ldots$

Comparison of Data Sources $\ldots \ldots \ldots \ldots \ldots \ldots \ldots \ldots \ldots \ldots \ldots \ldots$

Expected Cost of New Transmission Lines $\quad \ldots \ldots \ldots \ldots \ldots \ldots \ldots \ldots \ldots \ldots$

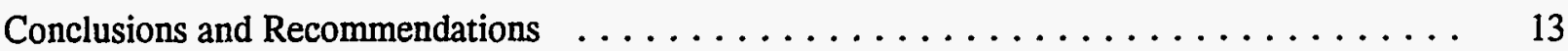

References $\ldots \ldots \ldots \ldots \ldots \ldots \ldots \ldots \ldots \ldots \ldots \ldots \ldots \ldots \ldots \ldots \ldots \ldots \ldots$

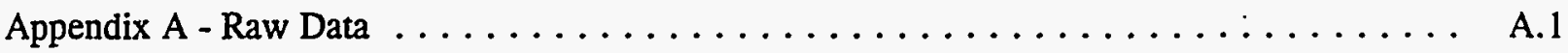

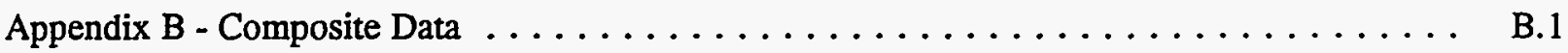

Appendix C - Adjusted FERC Data $\ldots \ldots \ldots \ldots \ldots \ldots \ldots \ldots \ldots \ldots \ldots \ldots \ldots \ldots \ldots$ 


\section{Figures}

1 Example of Data Smoothing Used to Create Composite Data Set $\ldots \ldots \ldots \ldots$

2 Curve Used to Determine Line Length Multiplier for Transmission Lines Shorter Than 20 Miles in Length $\ldots \ldots \ldots \ldots \ldots \ldots \ldots \ldots \ldots \ldots \ldots \ldots \ldots$

\section{Tables}

1 Mean Per-Mile Transmission Line Costs $\ldots \ldots \ldots \ldots \ldots \ldots \ldots \ldots$

2 Median Cost-Per-Mile Values for FERC Data Set by Voltage Level . . . . . . . . . . 9

3 Future Transmission Line Construction Costs $\ldots \ldots \ldots \ldots \ldots \ldots \ldots \ldots \ldots$ 


\section{Introduction}

The displacement or deferral of conventional AC transmission line installation is a key benefit associated with several technologies that are being developed with the support of the U.S. Department of Energy's Office of Energy Management (OEM). This includes HTS fault current limiters, batteries, SMES, and real-time system control, as well as HTS cable, HVDC, and high-phase-order transmission. In general, the value of displacement or deferral depends on the quantity of conventional AC transmission line affected, its unit capital cost, and the period of deferral.

This study addresses the unit capital cost of conventional AC transmission line. The preparation of Quality Metrics for OEM planning units identified a factor of five difference in the average cost per mile assumed for conventional AC transmission line. The unit cost of conventional AC transmission line does vary by a factor of 10 or more depending on the voltage level, conductor size, and many other design conditions. Therefore, all of the variation noted while preparing Quality Metrics could potentially be explained by different assumptions regarding the design conditions. Still, a factor of five difference across technology applications seems rather large when viewed from a national average perspective.

In response to this issue, the Pacific Northwest Laboratory (PNL), ${ }^{(a)}$ through its Management Analysis Program (MAP), conducted an investigation of transmission line cost data for OEM. The objective of this study was to develop a database for preparing preliminary estimates of transmission line costs based on the key design variables affecting cost. The database would provide a consistent set of cost assumptions for OEM, and other utility programs, that are applicable when conducting evaluations from a national or sitegeneric perspective. Care should be taken in applying the data in this report for site-specific analyses. In particular, land and environmental costs are highly variable and need to be carefully evaluated in any sitespecific study.

The balance of this report is divided into five sections that describe the sources of data, development of a composite database, comparison of data sources, calculation of average costs per mile, and conclusions and recommendations. Raw data are presented in Appendix A. The composite database recommended for preliminary estimating of conventional AC transmission line costs is presented in Appendix B. Adjusted FERC data used for comparison with the composite data are presented in Appendix C.

(a) Pacific Northwest Laboratory is operated for the U.S. Department of Energy by Battelle Memorial Institute under Contract DE-AC06-76RLO 1830. 


\section{-}




\section{Data Sources}

An extensive search of secondary information sources was conducted to determine if transmission line cost data already existed in a form useful to this study. While quite a few articles relevant to transmission line costs were identified, the scope of the articles or their orientation was not useful for this endeavor. Many articles dealt with site-specific costs or with the theoretical aspects of cost prediction or cost modeling.

In addition to the database searches, the following organizations were contacted to identify transmission line cost data:

- Electric Power Research Institute (EPRI)

- Energy Information Administration (EIA)

- Federal Energy Regulatory Commission (FERC)

- Edison Electric Institute (EEI)

- Oak Ridge National Laboratory (ORNL)

- Northwest Power Planning Council (NWPPC)

- California Public Utility Commission

- Oregon Public Utility Commission

- Washington Public Utility Commission

- Canadian Electric Association

- The Southern Company

- Tennessee Valley Authority (TVA)

- Southwestern Power Administration (SPA)

- Western Area Power Administration (WAPA)

- Bonneville Power Administration (BPA)

- Utility Data Institute (UDI).

Most of these organizations either did not collect the type of information sought, had site-specific data only, or only had data on transmission line component costs (e.g., conductor costs, cross-arm costs, etc.) as opposed to total transmission line costs based on actual design configurations. 
Both BPA and WAPA have extensive transmission line cost data based on their historical construction experience (BPA 1993; WAPA 1994). Each organization has maintained their respective database for a number of years, incorporating annual updates based on data from new installations, new vendor prices, and adjustments to account for inflation. These data are employed by BPA and WAPA to establish preliminary cost estimates for planned transmission line installations.

The WAPA data set contains cost estimates based on conductor size, voltage level, tower material type, tower frame type, and number of circuits. For combinations of these parameters that are actually used, average per-mile cost estimates are given. This data set is presented in Appendix A. The BPA data set contains the same type of information (per-mile cost estimates broken down by conductor size, voltage level, tower material type, tower frame type, and number of circuits). The BPA data set is presented in Appendix A as well.

There are a number of differences between the two data sets. In general, the BPA data set contains per-mile estimates for conductor sizes that are larger than those found in the WAPA data set. The WAPA data are normalized to flat terrain, while the BPA data exist for a variety of terrain types (flat, rolling, 50/50 [half rolling and half mountainous], and mountainous). The WAPA data set contains a separate table with additional cost components, expressed as percentages of principal contracts, that are intended to be added on to the per-mile figures shown (which are for principal contracts only) to arrive at total project cost. These additional cost components include planning, environmental, field data, land and rights, design and specifications, other contracts, and construction supervision. The BPA data have three of these cost components built into the per-mile estimates: field data, design and specifications, and principal contracts. An additional difference between the data sets is the approach for estimating the cost of land and land rights. This component is estimated as a percentage of principal contracts for the WAPA data, while fixed prices based on right-of-way width and geographic location are provided with the BPA data. Similarly, a range of costs is provided for the BPA data for environmental assessments, whereas this is one of the cost components expressed as a percentage of principal contracts for the WAPA data. Lastly, the per-mile figures contained in the raw BPA data set are expressed in 1993 dollars; those in the WAPA data set are in 1994 dollars.

Appendix A also contains transmission line cost data collected by FERC from investor-owned utilities for transmission lines constructed in 1992 (EIA 1994). Whereas the BPA and WAPA data represent federal power marketing agency costs, the FERC data covers costs incurred by private utilities. In addition, the BPA and WAPA data sets are based on experience in the Western United States, while the FERC data represent utilities from across the country.

The FERC data include "land," "structure," and "conductor" cost components, and the corresponding line length and voltage level, but the data do not specify detailed design parameters such as conductor size, number of circuits, or frame type. Therefore, the BPA and WAPA data sets were used to develop the composite data set; the FERC data were used for comparison purposes only. FERC staff indicated that while the intention is to collect data that includes all costs associated with transmission line construction, the data collection forms do not explicitly describe what is to be included with or excluded from each component. For comparison purposes, "land" was presumed to include land, land rights, and environmental costs, while all other costs were assumed to be included in the structure and conductor components. 


\section{Composite Database}

Several steps were taken to create a composite database from the BPA and WAPA data. These steps are outlined below, and the resulting composite database is presented in Appendix $B$. These data appear in Appendix B in two formats: as a table of the complete data set, and in tables with the data grouped by tower frame type, tower material type, and the number of circuits.

Each entry in the two databases was adjusted to reflect a common scope of hardware and services, and a common price year. For the BPA data, the per-mile dollar values were adjusted to 1994 dollars, using the Gross Domestic Product (GDP) implicit price deflator. The per-mile figures for the BPA data set were also adjusted to represent what the cost would have been for each configuration if it had been installed on flat terrain, using the appropriate terrain multiplier from the WAPA data set. For the WAPA data set, the original per-mile costs were increased to reflect the following components included in the BPA estimates: field data, design and specifications, and other contracts. These costs were added based on fixed percentages of principal contracts (the reference per-mile figures given in the WAPA data set), using percentages midway between the high and the low percentage figures provided with the WAPA data set.

The next step in creating a composite database was to generate per-mile estimates for each combination of parameters (voltage level, number of circuits, tower frame type, and tower material type) from the BPA and WAPA data that resulted in a "smooth" progression of per-mile cost with increasing conductor size. This was accomplished by fitting a line to the data using least squares regression techniques, with conductor size as the independent variable, and cost per-mile as the dependant variable. An example of this process appears in Figure 1. The example shown in Figure 1 is for $115-\mathrm{kV}$, single circuit, wood $\mathrm{H}$-frame lines. Appendix B contains per-mile estimates from the composite database for various combinations of design parameters.

Neither the BPA or WAPA data sets included estimated costs for $765-\mathrm{kV}$ transmission lines. The cost estimated for this voltage level was extrapolated from the existing data using the following approach. First, the mean cost per mile was calculated by voltage level, using the composite data set. These data appear in Table 1. The data for $115-\mathrm{kV}, 230-\mathrm{kV}, 345-\mathrm{kV}$, and $500-\mathrm{kV}$ transmission lines were extrapolated via linear regression, yielding an estimated average cost of $\$ 782,000$ per mile at $765-\mathrm{kV}$.

Table 1 contains the range as well as the mean per-mile cost estimates by voltage level based on the composite database. The range of per-mile costs at a given voltage level reflects variation in conductor size, tower material type, tower frame type, and number of circuits. 


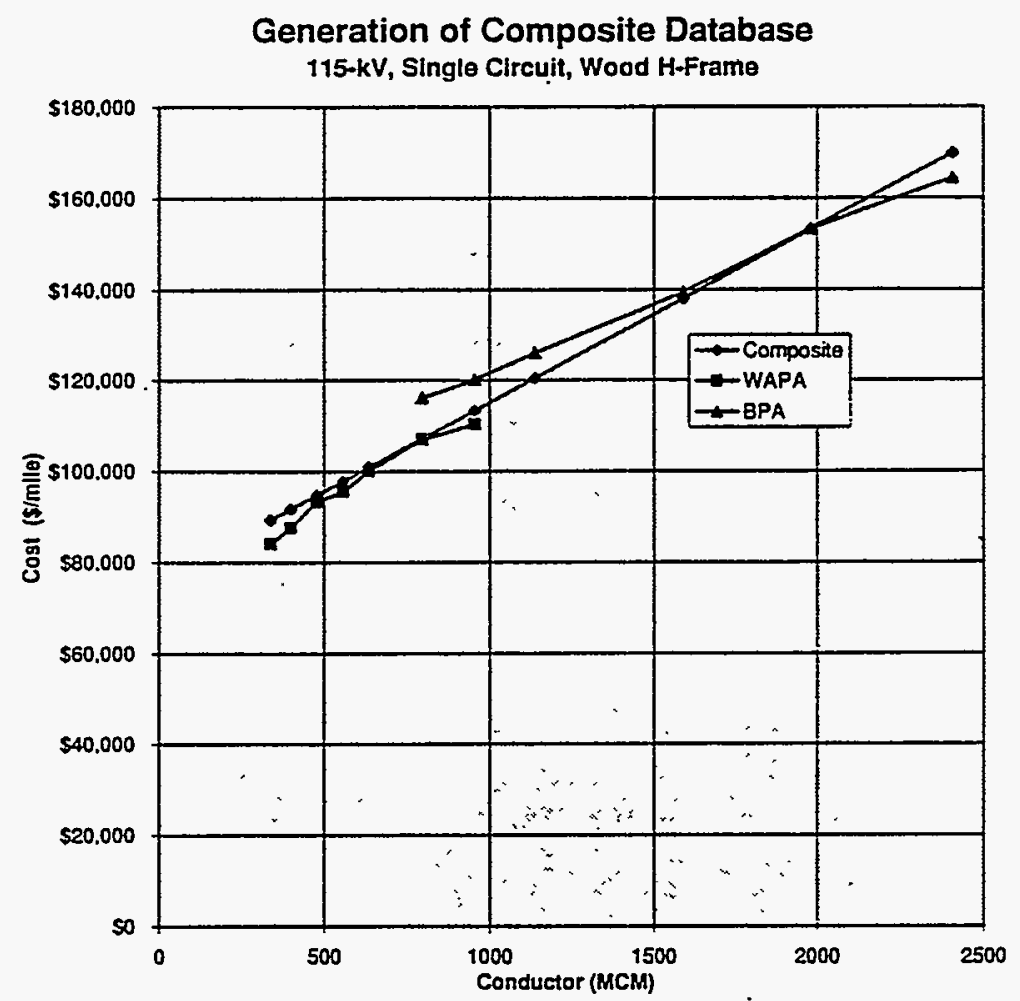

Figure 1. Example of Data Smoothing Used to Create Composite Data Set

Table 1. Mean Per-Mile Transmission Line Costs ${ }^{(a)}$

\begin{tabular}{|c|c|c||}
\hline Voltage (kV) & $\begin{array}{c}\text { Range of per-mile Costs } \\
(\$ 000)\end{array}$ & $\begin{array}{c}\text { Mean per-mile Cost from } \\
\text { Composite Database (\$000) }\end{array}$ \\
\hline 13.8 & $41-50$ & 44 \\
\hline 34.5 & $43-55$ & 48 \\
\hline 46 & $46-56$ & 49 \\
\hline 69 & $60-238$ & 107 \\
\hline 115 & $91-228$ & 124 \\
\hline 138 & $96-308$ & 165 \\
\hline 161 & $105-333$ & 182 \\
\hline 230 & $129-371$ & 235 \\
\hline 345 & $218-503$ & 341 \\
\hline 500 & $364-972$ & 519 \\
\hline 765 & Extrapolated & 782 \\
\hline
\end{tabular}


As noted previously, the composite database figures do not include land, environmental, planning, or construction supervision costs. These costs are estimated separately, based on a percentage of the composite database cost per-mile figure, using percentages suggested by WAPA. Alternatively, the land and environmental costs could be estimated using the fixed costs suggested by BPA. The specific land and environmental estimating rules of thumb suggested by WAPA and BPA are presented in Appendix B. Using these two approaches will yield slightly different results, as illustrated in the example below. Note that the per-mile estimates appearing in the composite database are for flat terrain. The appropriate terrain multiplier must be used for a given installation. The example shows how to calculate the total project cost for a given installation.

\section{Transmission Line: Installation: Project Cost Estimation}

This :example illustrates the approach used to calculate the total project cost for a transmission line installation ${ }_{r}$ first using the BPA and then the WAPA approaches to accounting for land and environmental costs. As with all of these data; this would serve only as a preliminary scoping estimate, which: would be followed by site-specific analyses.

Assume that a utility wants to install 20 miles of $230-\mathrm{kV}$, single-circuit line using lattice steel towers .1272 -conductor; over rolling terrain; with a 125-ft right-of-way through a non-urban area. The reference cost per-mile for the given design parameters is $\$ 183,000$, which can be found in Appendix. $B$. This figure would then be modified by the terrain:multiplier. For rolling terrain, this is $10 \%$ : Therefore; the adjusted cost is $\$ 183,000 \times 1.10=\$ 201,000$ per-mile. For 20 miles, the total cost.would be $\$ 4,020,000$.

Environmental, land, planning, and construction supervision costs are now added. Using the WAPA approach, a percentage of the reference cost-per-mile is added to account for these factors. With $6.5 \%$ for environmental, $5.5 \%$ for land, $0.75 \%$ for planning, and $7.5 \%$ for construction supervision, the grand total project cost would be $\$ 4,834,000$.

Using the BPA approach, fixed values for environmental and land costs are added. A mid-range price for environmental costs is $\$ 50,000$. For a 125-ft right-of-way through a non-urban area (averaging the Oregon and Washington values), the land cost would be $\$ 25,750$ per-mile, or $\$ 515,000$ for 20 miles. Adding in planning and construction supervision costs using the same percentages as with the WAPA approach, the grand total project cost would be $\$ 4,917,000$. 
An additional step is necessary to estimate the cost of transmission line installations less than 20 miles in length. Figure 2 shows the line length multiplier curve recommended by WAPA. The reference cost for a given configuration is multiplied by the value given by the curve for the length of the line. This is done prior to adding on environmental, land, planning, and construction supervision costs. The use of this curve is illustrated in the example below.

\footnotetext{
Using the same configuration as before, with a transmission line: only 10 miles long, the reference cost from Appendix $B$ is still $\$ 183,000$. Using Figure 2 , the line length multiplier for a 10-mile line is 1.7 . Therefore, the modified reference cost is $\$ 311,100$. For rolling terrain, this would be multiplied by 1.1 to obtain $\$ 342,210$ per mile. For 10 miles, the total cost would be $\$ 3,422,100$.
}

Adding in environmental; land, planning, and construction supervision costs as before yields a grand total project cost of $\$ 4,115,000$ using the WAPA approach, or $\$ 4,012,000$ using the BPA approach.

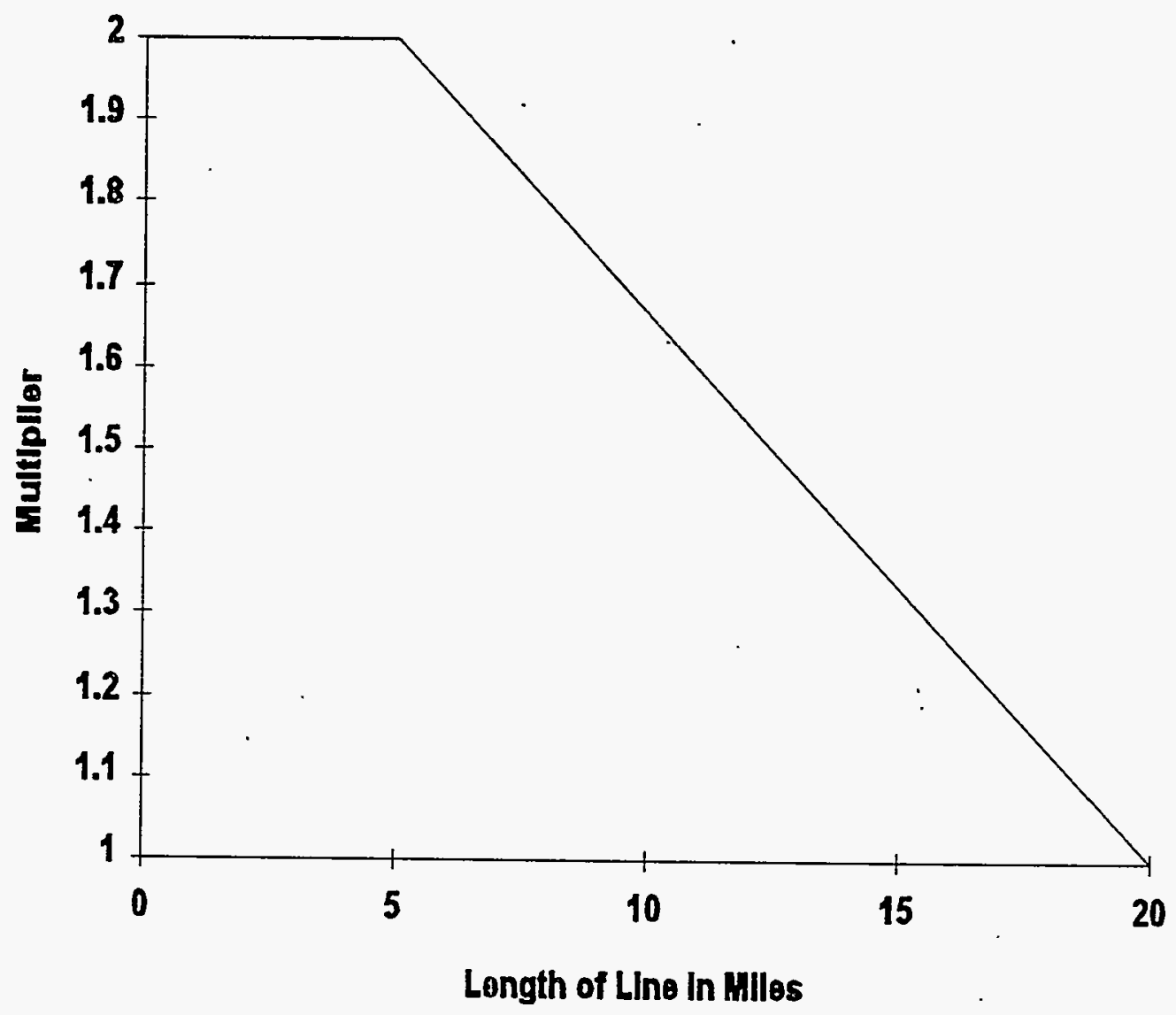

Figure 2. Curve Used to Determine Line Length Multiplier for Transmission Lines Shorter Than 20 Miles in Length 


\section{Comparison of Data Sources}

The FERC cost data were updated to 1994 dollars using the GDP implicit price deflator to be consistent with the composite data set. In addition, the per-mile costs of lines less than 20 miles long were multiplied by the appropriate value (line length factor) from Figure 2. The adjusted FERC data are presented in Appendix $\mathrm{C}$ for each utility.

The table in Appendix $C$ compares the adjusted structure and conductor cost for each line from the FERC data set to the range of values estimated by the composite (BPA and WAPA) data set for the same voltage level. A comparison of the FERC and composite data reveals that a few installations had per-mile costs outside of the corresponding composite data set range. However, the median of the FERC values for each voltage level lies within the composite data set range, as shown in Table 2. Note that the range of values from the composite data set is driven by explicit variations in design configurations, while the FERC data set range results from unspecified configurations that are likely different. Therefore, the results of the comparison must be used cautiously.

An examination of "land" costs reported in the FERC data set revealed a median cost equal to $13 \%$ of the combined structure and conductor cost. This is consistent with the WAPA guideline (9-15\%) for estimating land and environmental costs as a fraction of hardware-related costs, assuming that the FERC "land" component includes environmental-related costs as well as land and right-of-way costs, as was discussed in the Data Sources Section.

Land and environmental costs are extremely site-specific. Additional data collection and analysis of these two cost components would clarify the variability that exists. However, BPA, WAPA, and FERC data all indicate that land and environmental costs, on average, represent less than $20 \%$ of the total cost. Therefore, further analysis of these two cost components should probably be tempered.

Comparison of FERC and composite (BPA and WAPA) data sets identified no significant differences. Therefore, the composite data set appears to be a reasonable proxy for estimating national average transmission line costs.

Table 2. Median Cost-Per-Mile Values for FERC Data Set by Voltage Level

\begin{tabular}{|c|c|c|c||}
\hline $\begin{array}{c}\text { Voltage Level } \\
(\mathrm{kV})\end{array}$ & $\begin{array}{c}\text { FERC Median } \\
\text { Cost-per-Mile } \\
\mathbf{( \$ 0 0 0 )}\end{array}$ & $\begin{array}{c}\text { FERC Cost-per- } \\
\text { Mile Range } \\
\mathbf{( \$ 0 0 0 )}\end{array}$ & $\begin{array}{c}\text { Range of Values from } \\
\text { Composite BPA/WAPA } \\
\text { Data Set (\$000) }\end{array}$ \\
\hline 138 & 161 & $17-1787$ & $96-308$ \\
\hline 230 & 202 & $51-1356$ & $129-371$ \\
\hline 345 & 406 & $92-440$ & $218-503$ \\
\hline
\end{tabular}





\section{Expected Cost of New Transmission Lines}

The North American Electric Reliability Council (NERC) provides estimates of the number of miles of new transmission lines expected to be constructed by 2002 for $230-\mathrm{kV}$ and higher voltage (NERC 1993). These data were used to estimate the total cost of this new construction and the weighted (by expected future construction mileage) average cost per mile. These figures appear in Table 3.

In Table 3, the average cost per-mile by voltage level (from Table 1) was first multiplied by 1.1 to adjust to rolling terrain, and then multiplied by the number of miles NERC estimates to be added by 2002. Land, environmental, planning, and construction supervision costs are added to the base cost figures using the average percentage multipliers $(5.5 \%, 6.5 \%, 0.75 \%$, and $7.5 \%$, respectively) suggested by WAPA. This yields the grand totals shown.

Table 3. Future Transmission Line Construction Costs

\begin{tabular}{|c|c|c|c|c|c|}
\hline Voltage Level (kV) & 230 & 345 & 500 & 765 & $\begin{array}{l}\text { Weighted Average Cost } \\
\text { (by Number of Miles) }\end{array}$ \\
\hline $\begin{array}{l}\text { Base Cost per-mile } \\
(\$ 000)\end{array}$ & 258 & 376 & 571 & 861 & 417 \\
\hline $\begin{array}{l}\text { Number of Additional } \\
\text { Miles }\end{array}$ & $-4,365$ & 2,452 & 4,929 & 115 & N/A \\
\hline $\begin{array}{l}\text { Total Base Cost } \\
(\$ 000,000)\end{array}$ & 1,128 & 921 & 2,758 & 99 & N/A \\
\hline Land $(\$ 000)$ & 62 & 51 & 152 & 5 & $\mathrm{~N} / \mathrm{A}$ \\
\hline Environmental $(\$ 000)$ & 73 & 60 & 179 & 6 & N/A \\
\hline Planning $(\$ 000)$ & 8 & 7 & 21 & 1 & N/A \\
\hline $\begin{array}{l}\text { Construction } \\
\text { Supervision }(\$ 000)\end{array}$ & 85 & 69 & 207 & 7 & N/A \\
\hline $\begin{array}{l}\text { Grand Total } \\
(\$ 000,000)\end{array}$ & 1,356 & 1,107 & 3,316 & 119 & N/A \\
\hline $\begin{array}{l}\text { Grand Total per Mile } \\
(\$ 000)\end{array}$ & 311 & 452 & 687 & 1,035 & 502 \\
\hline
\end{tabular}




\section{Conclusions and Recommendations}

Current transmission line stock and projected capacity additions are usually measured by the number of miles at each of the principal voltage levels. Therefore, the composite database and discussions of transmission line costs are focused on this design variable. However, the other cost drivers included in the BPA and WAPA data sets, namely conductor size, terrain type, tower frame type, tower material type, and number of circuits, could also have been used as classification variables to group per-mile costs. In particular, conductor size would appear to be the single most significant cost driver. The result is that reported costs per mile for a given voltage level vary significantly, but can be explained by these additional design variables, if they are known.

The BPA and WAPA data sets employed in this analysis both represent transmission line costs incurred by federal power marketing agencies in the Western United States. No significant differences were identified when these data sets were compared to the FERC data collected from investor-owned utilities from across the country. Therefore, the composite data set appears to be a reasonable proxy for estimating national average costs.

The extremely site-specific nature of environmental and land (right-of-way) costs make it very difficult to estimate the costs for these components in a generalized manner. Additional data collection and analysis of the costs for these two components would develop a better understanding of the variability that exists. However, BPA, WAPA, and FERC data indicate that these two components typically combine to less than $20 \%$ of the total cost, so only a modest analytical effort is recommended. 


\section{-}




\section{References}

Bonneville Power Administration (BPA). 1993. "Internal Memorandum: Transmission Line Estimating Data." Portland, Oregon.

Energy Information Administration (EIA). 1994. Electric Trade in the United States 1992. DOE/EIA-0531(92), Washington, D.C.

North American Electric Reliability Council (NERC). 1993. Electricity Supply and Demand 1993-2002. Princeton, New Jersey.

Western Area Power Administration (WAPA). 1994. Conceptual Planning and Budget Cost Estimating Guide. Golden, Colorado. 
Appendix A

Raw Data 


\section{Appendix A}

\section{Raw Data}

This appendix contains the raw data provided by Bonneville Power Administration (BPA) or Western Area Power Administration (WAPA), as indicated in the "Agency" column. The original costs provided by BPA were adjusted from 1993 to 1994 dollars and to reflect Flat terrain. The original costs provided by WAPA were adjusted to include Field Data, Design and Specifications, and Other Contracts. The "Adjusted" costs were used to develop the composite data set presented in Appendix B.

The second table of this appendix presents the raw data collected by Federal Energy Regulatory Commission (FERC) from investor-owned utilities. The adjusted FERC data are presented in Appen$\operatorname{dix}$ C. 
Table A.1. RAW Data

Terrain Lookup Table - Provided with WAPA data.

\begin{tabular}{|cc|cc|}
\hline Fiat & Rolling & $50 / 50$ & MoLntaindus \\
\hline $0 \%$ & $10 \%$ & $18 \%$ & $25 \%$ \\
\hline
\end{tabular}

Other Factors (as percent additlons to base cost) - Provided with WAPA data.

\begin{tabular}{|lcc|}
\hline Cost & Low & High \\
\hline Planning & $0.5 \%$ & $1.0 \%$ \\
Environmental & $5.0 \%$ & $8.0 \%$ \\
Field Data & $5.0 \%$ & $10.0 \%$ \\
Land and Rights & $4.0 \%$ & $7.0 \%$ \\
Design \& Specifications & $0.5 \%$ & $1.0 \%$ \\
Oiner Contracts & $4.0 \%$ & $7.0 \%$ \\
Construction Supervision & $5.0 \%$ & $10.0 \%$ \\
\hline
\end{tabular}

\begin{tabular}{|c|c|c|c|c|c|c|c|c|}
\hline RAW Cost & Adjusted Cost & Voltage $(k V)$ & Conductor Size & $\begin{array}{c}\text { Number of } \\
\text { Circults }\end{array}$ & $\begin{array}{c}\text { Terrain } \\
\text { Type }\end{array}$ & Pole Type & Frame Type & Agency \\
\hline$\$ 36,000$ & $\$ 40,950$ & 13.8 & $1 / 0$ & 1 & Flat & Wood & Single Pole & WAPA \\
\hline$\$ 37,000$ & $\$ 42,088$ & 13.8 & $2 / 0$ & 1 & Flat & Wood & Single Pole & WAPA \\
\hline$\$ 38,000$ & $\$ 43,225$ & 13.8 & $3 / 0$ & 1 & Fiat & Wood & Single Pole & WAPA \\
\hline$\$ 40,000$ & $\$ 45,500$ & 13.8 & $4 / 0$ & 1 & Flat & Wood & Single Pole & WAPA \\
\hline$\$ 42.000$ & $\$ 47,775$ & 13.8 & 266.8 & 1 & Flat & Wood & Single Pole & WAPA \\
\hline$\$ 44,000$ & $\$ 50,050$ & 13.8 & 336.4 & 1 & Flat & Wood & Single Pole & WAPA \\
\hline$\$ 38,000$ & $\$ 43,225$ & 34.5 & $1 / 0$ & 1 & Flat & Wood & Single Pole & WAPA \\
\hline$\$ 40,000$ & $\$ 45,500$ & 34.5 & $2 / 0$ & 1 & Flat & Wood & Single Pole & WAPA \\
\hline$\$ 41,000$ & $\$ 46,638$ & 34.5 & $3 / 0$ & 1 & Flat & Wood & Single Pole & WAPA \\
\hline$\$ 42,000$ & $\$ 47,775$ & 34.5 & $4 / 0$ & 1 & Flat & Wood & Single Pole & WAPA \\
\hline$\$ 44,000$ & $\$ 50,050$ & 34.5 & 266.8 & 1 & Fiat & Wood & Single Pole & WAPA \\
\hline$\$ 48,000$ & $\$ 54,600$ & 34.5 & 336.4 & 1 & Fiat & Wood & Single Pole & WAPA \\
\hline$\$ 40,000$ & $\$ 45,500$ & 46 & $1 / 0$ & 1 & Flat & Wood & Single Pole & WAPA \\
\hline$\$ 41,000$ & $\$ 46,638$ & 46 & $2 / 0$ & 1 & Flat & Wood & Single Pole & WAPA \\
\hline$\$ 42,000$ & $\$ 47,775$ & 46 & $3 / 0$ & 1 & Flat & Wood & Single Pole & WAPA \\
\hline$\$ 43,000$ & $\$ 48,913$ & 46 & $4 / 0$ & 1 & Flat & Wood & Single Pole & WAPA \\
\hline$\$ 46,000$ & $\$ 52,325$ & 46 & 266.8 & 1 & Flat & Wood & Single Pole & WAPA \\
\hline$\$ 49,000$ & $\$ 55,738$ & 46 & 336.4 & 1 & Flat & Wood & Single Pole & WAPA \\
\hline$\$ 53,000$ & $\$ 60,288$ & 69 & $4 / 0$ & 1 & Flat & Wood & Single Pole & WAPA \\
\hline$\$ 55,000$ & $\$ 62,563$ & 69 & 266.8 & 1 & Flat & Wood & Single Pole & WAPA \\
\hline$\$ 58,000$ & $\$ 65,975$ & 69 & 336.4 & 1 & Flat & Wood & Single Pole & WAPA \\
\hline$\$ 60,000$ & $\$ 68,250$ & 69 & 397.5 & 1 & Flat & Wood & Single Pole & WAPA \\
\hline$\$ 64,000$ & $\$ 72,800$ & 69 & 477 & 1 & Flat & Wood & Single Pole & WAPA \\
\hline$\$ 66,000$ & $\$ 75,075$ & 69 & 556.5 & 1 & Flat & Wood & Single Pole & WAPA \\
\hline$\$ 70,000$ & $\$ 79,625$ & 69 & 636 & 1 & Flat & Waod & Single Pole & WAPA \\
\hline$\$ 76.000$ & $\$ 86,450$ & 69 & 795 & 1 & Flat & Wood & Single Pole & WAPA \\
\hline$\$ 60,000$ & $\$ 68,250$ & 69 & $4 / 0$ & 1 & Fiat & Wood & H-Frame & WAPA \\
\hline$\$ 62,000$ & $\$ 70,525$ & 69 & 266.8 & 1 & Flat & Wood & H.Frame & WAPA \\
\hline$\$ 65,000$ & $\$ 73,938$ & 69 & 336.4 & 1 & Flat & Wood & H-Frame & WAPA \\
\hline$\$ 67,000$ & $\$ 76,213$ & 69 & 397.5 & 1 & Flat & Wood & H-Frame & WAPA \\
\hline$\$ 71,000$ & $\$ 80,763$ & 69 & 477 & 1 & Flat & Wood & H-Frame & WAPA \\
\hline$\$ 73,000$ & $\$ 83,038$ & 69 & 556.5 & 1 & Flat & Wood & H-Frame & WAPA \\
\hline$\$ 77,000$ & $\$ 87,588$ & 69 & 636 & 1 & Flat & Wood & H-Frame & WAPA \\
\hline$\$ 84,000$ & $\$ 95,550$ & 69 & 795 & 1 & Flat & Wood & H.Frame & WAPA \\
\hline$\$ 89,000$ & $\$ 101,238$ & 69 & 397.5 & 1 & Flat & Steel & Single Pole & WAPA \\
\hline$\$ 93,000$ & $\$ 105,788$ & 69 & 477 & 1 & Flat & Steel & Single Pole & WAPA \\
\hline$\$ 96,000$ & $\$ 109,200$ & 69 & 556.5 & 1 & Flat & Steel & Single Pole & WAPA \\
\hline$\$ 100,000$ & $\$ 113,750$ & 69 & 636 & 1 & Flat & Sieel & Single Pole & WAPA \\
\hline$\$ 105,000$ & $\$ 119,438$ & 69 & 795 & 1 & Flat & Steel & SIngle Pole & WAPA \\
\hline$\$ 108,000$ & $\$ 122,850$ & 69 & 954 & 1 & Flat & Steel & Single Pole & WAPA \\
\hline$\$ 113,000$ & $\$ 128,538$ & 69 & 1272 & 1 & Flat & Steel & Single Pole & WAPA \\
\hline$\$ 129.000$ & $\$ 146,738$ & 69 & 397.5 & 2 & Flat & Steel ${ }^{-}$ & Single Pole & WAPA \\
\hline$\$ 141,000$ & $\$ 160.388$ & 69 & 477 & 2 & Flat & Steel & Single Pole & WAPA \\
\hline$\$ 150,000$ & $\$ 170,625$ & 69 & 556.5 & 2 & Flat & Steel & Single Pole & WAPA \\
\hline$\$ 164,000$ & $\$ 186,550$ & 69 & 636 & 2 & Flat & Steel & Single Pole & WAPA \\
\hline$\$ 180,000$ & $\$ 204.750$ & 69 & 795 & 2 & Flat & Steel & Single Pole & WAPA \\
\hline
\end{tabular}


Table A.1. (contd)

\begin{tabular}{|c|c|c|c|c|c|c|c|c|}
\hline RAW Cost & Adjusted Cost & Voltage (kV) & Conductor Size & $\begin{array}{c}\text { Number of } \\
\text { Clrcults }\end{array}$ & $\begin{array}{c}\text { Terrain } \\
\text { Type }\end{array}$ & Pole Type & Frame Type & Agency \\
\hline$\$ 192,000$ & $\$ 218,400$ & 69 & 954 & 2 & Flat & Steel & Single Pole & WAPA \\
\hline$\$ 209,000$ & $\$ 237.738$ & 69 & 1272 & 2 & Flat & Steel & Single Pole & WAPA \\
\hline$\$ 109,900$ & $\$ 102,483$ & 115 & 398 & 1 & Rolling & Wood & Single Pole & BPA \\
\hline$\$ 119,740$ & $\$ 111,659$ & 115 & 795 & 1 & Rolling & Wood & Single Pole & BPA \\
\hline$\$ 133,260$ & $\$ 124,267$ & 115 & 1137 & 1 & Rolling & Wood & Single Pole & BPA \\
\hline$\$ 151,500$ & $\$ 141,276$ & 115 & 1589 & 1 & Rolling & Wood & Single Pole & BPA \\
\hline$\$ 124,490$ & $\$ 116,089$ & 115 & 795 & 1 & Rolling & Wood & H-Frame & BPA \\
\hline$\$ 128,810$ & $\$ 120,117$ & 115 & 954 & 1 & Rolling & Wood & H-Frame & BPA \\
\hline$\$ 135,200$ & $\$ 126,076$ & 115 & 1137 & 1 & Rolling & Wood & H-Frame & BPA \\
\hline$\$ 149,520$ & $\$ 139,429$ & 115 & 1589 & 1 & Rolling & Wood & H-Frame & BPA \\
\hline$\$ 164,260$ & $\$ 153,175$ & 115 & 1979 & 1 & Rolling & Wood & H-Frame & BPA \\
\hline$\$ 176,250$ & $\$ 164,356$ & 115 & 2406 & 1 & Rolling & Wood & H-Frame & BPA \\
\hline$\$ 140,000$ & $\$ 130,552$ & 115 & 795 & 1 & Rolling & Steel & Single Pole & BPA \\
\hline$\$ 191,720$ & $\$ 166,661$ & 115 & 795 & 1 & $50 / 50$ & Steel & Lattice & BPA \\
\hline$\$ 262,730$ & $\$ 228,389$ & 115 & 795 & 2 & $50 / 50$ & Steel & Lattice & BPA \\
\hline$\$ 71,000$ & $\$ 80,763$ & 115 & 397.5 & 1 & Flat & Wood & Single Pole & WAPA \\
\hline$\$ 74,000$ & $\$ 84,175$ & 115 & 477 & 1 & Flat & Wood & SIngle Pole & WAPA \\
\hline$\$ 77.000$ & $\$ 87,588$ & 115 & 556.5 & 1 & Flat & Wood & Single Pole & WAPA \\
\hline$\$ 82,000$ & $\$ 93,275$ & 115 & 636 & 1 & Flat & Wood & Single Pole & WAPA \\
\hline$\$ 88,000$ & $\$ 100,100$ & 115 & 795 & 1 & Flat & Wood & Single Pole & WAPA \\
\hline$\$ 74,000$ & $\$ 84,175$ & 115 & 336.4 & 1 & Flat & Wood & H-Frame & WAPA \\
\hline$\$ 77,000$ & $\$ 87,588$ & 115 & 397.5 & 1 & Flat & Wood & H-Frame & WAPA \\
\hline$\$ 82,000$ & $\$ 93,275$ & 115 & 477 & 1 & Flat & Wood & H-Frame & WAPA \\
\hline$\$ 84,000$ & $\$ 95,550$ & 115 & 556.5 & 1 & Flat & Wood & H-Frame & WAPA \\
\hline$\$ 88,000$ & $\$ 100,100$ & 115 & $\varpi 6$ & 1 & Flat & Wood & H-Frame & WAPA \\
\hline$\$ 94,000$ & $\$ 106,925$ & 115 & 795 & 1 & Flat & Wood & H-Frame & WAPA \\
\hline$\$ 97,000$ & $\$ 110,338$ & 115 & 954 & 1 & Flat & Wood & H-Frame & WAPA \\
\hline$\$ 109,000$ & $\$ 123,988$ & 115 & 477 & 1 & Flat & Steel & Single Pole & WAPA \\
\hline$\$ 111,000$ & $\$ 126,263$ & 115 & 556.5 & 1 & Flat & Steel & Single Pole & WAPA \\
\hline$\$ 114,000$ & $\$ 129,675$ & 115 & 636 & 1 & Flat & Steel & Single Pole & WAPA \\
\hline$\$ 120,000$ & $\$ 136,500$ & 115 & 795 & 1 & Flat & Sieel & Single Pole & WAPA \\
\hline$\$ 123,000$ & $\$ 139,913$ & 115 & 954 & 1 & Flat & Steel & Single Pole & WAPA \\
\hline$\$ 130,000$ & $\$ 147,875$ & 115 & 1272 & 1 & Flat & Steel & Single Pole & WAPA \\
\hline$\$ 142,000$ & $\$ 161,525$ & 115 & 397.5 & 2 & Flat & Steel & Single Pole & WAPA. \\
\hline$\$ 159,000$ & $\$ 180,863$ & 115 & 477 & 2 & Flat & Steel & Single Pole & WAPA \\
\hline$\$ 173,000$ & $\$ 196,788$ & 115 & 556.5 & 2 & Flat & Steel & Single Pole & WAPA \\
\hline$\$ 190,000$ & $\$ 216,125$ & $\$ 15$ & 636 & 2 & Flat & Steel & Single Pole & WAPA \\
\hline$\$ 212,000$ & $\$ 241,150$ & 115 & 795 & 2 & Flat & Steel & Single Pole & WAPA \\
\hline$\$ 228,000$ & $\$ 259,350$ & 115 & 954 & 2 & Flat & Steel & Single Pole & WAPA \\
\hline$\$ 250,000$ & $\$ 284,375$ & 115 & 1272 & 2 & Flat & Steel & Single Pole & WAPA \\
\hline$\$ 84,000$ & $\$ 95,550$ & 138 & 397.5 & 1 & Flat & Wood & H-Frame & WAPA \\
\hline$\$ 88,000$ & $\$ 100,100$ & 138 & 477 & 1 & Flat & Wood & H-Frame & WAPA \\
\hline$\$ 90,000$ & $\$ 102,375$ & 138 & 556.5 & 1 & Flat & Wood & H-Frame & WAPA \\
\hline$\$ 94,000$ & $\$ 106,925$ & 138 & 636 & 1 & Flat & Wood & H-Frame & WAPA \\
\hline$\$ 100,000$ & $\$ 113,750$ & 138 & 795 & 1 & Flat & Wood & H-Frame & WAPA \\
\hline$\$ 103,000$ & $\$ 117,163$ & 138 & 954 & 1 & Flat & Wood & H-Frame & WAPA \\
\hline$\$ 111,000$ & $\$ 126,263$ & 138 & 477 & 1 & Flat & Steel & Single Pole & WAPA \\
\hline$\$ 116,000$ & $\$ 131,950$ & 138 & 556.5 & 1 & Flat & Steel & Single Pole & WAPA \\
\hline$\$ 121,000$ & $\$ 137,638$ & 138 & 636 & 1 & Flat & Steel & Single Pole & WAPA \\
\hline$\$ 130,000$ & $\$ 147,875$ & 138 & 795 & 1 & Flat & Steel & Single Pole & WAPA \\
\hline$\$ 135,000$ & $\$ 153,563$ & 138 & 954 & 1 & Flat & Steel & Single Pole & WAPA \\
\hline$\$ 143,000$ & $\$ 162,663$ & 138 & 1272 & 1 & Flar & Sieel & Single Pole & WAPA \\
\hline$\$ 168,000$ & $\$ 191,100$ & 138 & 477 & 2 & Flat & Steel & Single Pole & WAPA \\
\hline$\$ 185,000$ & $\$ 210,438$ & 138 & 556.5 & 2 & Flat & Steel & Single Pole & WAPA \\
\hline$\$ 204,000$ & $\$ 232,050$ & 138 & 636 & 2 & Flat & Steel & Single Pole & WAPA \\
\hline$\$ 227,000$ & $\$ 258,213$ & 138 & 795 & 2 & Flat & Steel & Single Pole & WAPA \\
\hline$\$ 247,000$ & $\$ 280,963$ & 138 & 954 & 2 & Flat & Steel & Single Pole & WAPA \\
\hline$\$ 271,000$ & $\$ 308,263$ & 138 & 1272 & 2 & Flat & Steel & Single Pole & WAPA \\
\hline$\$ 92,000$ & $\$ 104,650$ & 161 & 477 & 1 & Flat & Wood & H-Frame & WAPA \\
\hline$\$ 95,000$ & $\$ 108,063$ & 161 & 556.5 & 1 & Flat & Wood & H-Frame & WAPA \\
\hline$\$ 98,000$ & $\$ 111,475$ & 161 & 636 & 1 & Flat & Wood & H-Frame & WAPA \\
\hline$\$ 104,000$ & $\$ 118,300$ & 161 & 795 & 1 & Flat & Wood & H-Frame & WAPA \\
\hline$\$ 108,000$ & $\$ 122,850$ & 164 & 954 & 1 & Flat & Wood & H-Frame & WAPA \\
\hline$\$ 114,000$ & $\$ 129,675$ & 161 & 477 & 1 & Flat & Steel & Single Pole & WAPA \\
\hline$\$ 121,000$ & $\$ 137,638$ & 161 & .556 .5 & 1 & Flat & Steel & Single Pole & WAPA \\
\hline$\$ 131,000$ & $\$ 149,013$ & 161 & 636 & 1 & Flat & Steel & Single Pole & WAPA \\
\hline$\$ 141,000$ & $\$ 160,388$ & 161 & 795 & 1 & Flat & Steel & Single Pole & WAPA \\
\hline
\end{tabular}


Table A.1. (contd)

\begin{tabular}{|c|c|c|c|c|c|c|c|c|}
\hline RAW Cost & Adjusted Cost & Voltage (kV) & Conduatór Size & $\begin{array}{l}\text { Number bf } \\
\text { Circuils }\end{array}$ & $\begin{array}{c}\text { Terraln } \\
\text { Type }\end{array}$ & Pole Type & Frame Type & Agency \\
\hline$\$ 149,000$ & $\$ 169,488$ & 161 & 954 & 1 & Flat & Steel & Single Pole & WAPA \\
\hline$\$ 160,000$ & $\$ 182,000$ & 161 & 1272 & 1 & Flat & Steel & Single Pole & WAPA \\
\hline$\$ 182,000$ & $\$ 207,025$ & 161 & 477 & 2 & Flat & Steel & Single Pole & WAPA \\
\hline$\$ 201,000$ & $\$ 228,638$ & 161 & 556.5 & 2 & Flat & Steel & Single Pole & WAPA \\
\hline$\$ 222,000$ & $\$ 252,525$ & 161 & 636 & 2 & Flat & Steel & Single Pole & WAPA \\
\hline$\$ 247,000$ & $\$ 280,963$ & 161 & 795 & 2 & Flat & Steel & Single Pole & WAPA \\
\hline$\$ 268,000$ & $\$ 304,850$ & 161 & 954 & 2 & Flat & Steel & Single Pole & WAPA \\
\hline$\$ 293,000$ & $\$ 333,288$ & 161 & 1272 & 2 & Flat & Steel & Single Pole & WAPA \\
\hline$\$ 133,080$ & $\$ 124.099$ & 230 & 795 & 1 & Rolling & Wood & H-Frame & BPA \\
\hline$\$ 137.100$ & $\$ 127.848$ & 230 & 954 & 1 & Rolling & Wood & H-Frame & BPA \\
\hline$\$ 144,730$ & $\$ 134,963$ & 230 & 1137 & 1 & Rolling & Wood & H-Frame & BPA \\
\hline$\$ 159,830$ & $\$ 149,044$ & 230 & 1589 & 1 & Rolling & Wood & $H$-Frame & BPA \\
\hline$\$ 174,230$ & $\$ 162,472$ & 230 & 1979 & 1 & Rolling & Wood & H-Frame & BPA \\
\hline$\$ 187,480$ & $\$ 174,828$ & 230 & 2406 & 1 & Rolling & Wood & H-Frame & BPA \\
\hline$\$ 172.220$ & $\$ 160,597$ & 230 & 993 & 1 & Rolling & Steel & Lantice & BPA \\
\hline$\$ 176,980$ & $\$ 165,036$ & 230 & 1114 & 1 & Rolling & Steel & Lanice & BPA \\
\hline$\$ 229,000$ & $\$ 213,546$ & 230 & 1509 & 1 & Rolling & Steel & Lattice & BPA \\
\hline$\$ 235,960$ & $\$ 220,036$ & 230 & 1917 & 1 & Rolling & Steel & Lattice & BPA \\
\hline$\$ 252,830$ & $\$ 235,767$ & 230 & 2406 & 1 & Rolling & Steel & Lattice & BPA \\
\hline$\$ 212,250$ & $\$ 184,507$ & 230 & 993 & 1 & $50 / 50^{\circ}$ & Steel & Lattice & BPA \\
\hline$\$ 217,320$ & $\$ 188,915$ & 230 & 1114 & 1 & $50 / 50$ & Steel & Laltice & BPA \\
\hline$\$ 261,860$ & $\$ 227,633$ & 230 & 1509 & 1 & $50 / 50$ & Steel & Lattice & BPA \\
\hline$\$ 268,720$ & $\$ 233,596$ & 230 & 1917 & 1 & $50 / 50$ & Steel & Lattice & BPA \\
\hline$\$ 285,250$ & $\$ 247,966$ & 230 & 2406 & 1 & $50 / 50$ & Steel & Lattice & BPA \\
\hline$\$ 236,240$ & $\$ 220,297$ & 230 & 1589 & 2 & Rolling & Wood & H-Frame & BPA \\
\hline$\$ 310,480$ & $\$ 289,527$ & 230 & 1114 & 2 & Rolling & Steel & Single Pole & BPA \\
\hline$\$ 358,670$ & $\$ 334,465$ & 230 & 1589 & 2 & Rolling & Steel & Single Pole & BPA \\
\hline$\$ 285,390$ & $\$ 266,130$ & 230 & 993 & 2 & Rolling & Steel & Lattice & BPA \\
\hline$\$ 293,840$ & $\$ 274.010$ & 230 & 1114 & 2 & Rolling & Steel & Lattice & BPA \\
\hline$\$ 344,200$ & $\$ 320,971$ & 230 & 1509 & 2 & Rolling & Steel & Lattice & BPA \\
\hline$\$ 357.690$ & $\$ 333,551$ & 230 & 1917 & 2 & Rolling & Steel & Lattice & BPA \\
\hline$\$ 382,570$ & $\$ 356,752$ & 230 & 2406 & 2 & Rolling & Steel & Lattice & BPA \\
\hline$\$ 333,200$ & $\$ 289,648$ & 230 & 993 & 2 & $50 / 50^{\circ}$ & Steel & Lattice & BPA \\
\hline$\$ 342,360$ & $\$ 297,611$ & 230 & 1114 & 2 & $50 / 50$ & Steel & Lantice & BPA \\
\hline$\$ 376,160$ & $\$ 326,993$ & 230 & 1509 & 2 & $50 / 50$ & Steel & Lattice & BPA \\
\hline$\$ 389,650$ & $\$ 338,720$ & 230 & 1917 & 2 & $50 / 50$ & Steel & Lattice & BPA \\
\hline$\$ 415,210$ & $\$ 360,939$ & 230 & 2406 & 2 & $50 / 50$ & Steel & Lanice & BPA \\
\hline$\$ 119,000$ & $\$ 135,363$ & 230 & 795 & 1 & Flat & Wood & H-Frame & WAPA \\
\hline$\$ 122,000$ & $\$ 138,775$ & 230 & 954 & 1 & Flat & Wood & H-Frame & WAPA \\
\hline$\$ 128,000$ & $\$ 145,600$ & 230 & 1272 & 1 & Flat & Wood & H-Frame & WAPA \\
\hline$\$ 123,000$ & $\$ 139,913$ & 230 & 477 & 1 & Flat & Steel & Single Pole & WAPA \\
\hline$\$ 133,000$ & $\$ 151,288$ & 230 & 556.5 & 1 & Flat & Steel & Single Pole & WAPA \\
\hline$\$ 143,000$ & $\$ 162,663$ & 230 & 636 & 1 & Flat & Steel & SIngle Pole & WAPA \\
\hline$\$ 155,000$ & $\$ 176,313$ & 230 & 795 & 1 & Flat & Steel & SIngle Pole & WAPA \\
\hline$\$ 166,000$ & $\$ 188,825$ & 230 & 954 & 1 & Flat & Steel & Single Pole & WAPA \\
\hline$\$ 179,000$ & $\$ 203,613$ & 230 & 1272 & 1 & Flat & Steel & Single.Pole & WAPA \\
\hline$\$ 207,000$ & $\$ 235,463$ & 230 & 477 & 2 & Flat & Steel & Single Pole & WAPA \\
\hline$\$ 222,000$ & $\$ 252,525$ & 230 & 556.5 & 2 & Flat & Steel & Single Pole & WAPA \\
\hline$\$ 240,000$ & $\$ 273,000$ & 230 & 636 & 2 & Flat & Steel & Single Pole & WAPA \\
\hline$\$ 261,000$ & $\$ 296,888$ & 230 & 795 & 2 & Flat & Steel & Single Pole & WAPA \\
\hline$\$ 280,000$ & $\$ 318,500$ & 230 & 954 & 2 & Flat & Steel & Single Pole & WAPA \\
\hline$\$ 302,000$ & $\$ 343,525$ & 230 & 1272 & 2 & Flat & Steel & Single Pole & WAPA \\
\hline$\$ 119,000$ & $\$ 135,363$ & 230 & 795 & 1 & Flat & Steel & Lattice & WAPA \\
\hline$\$ 132,000$ & $\$ 150,150$ & 230 & 954 & 1 & Flat & Steel & Lattice & WAPA \\
\hline$\$ 150,000$ & $\$ 170,625$ & 230 & 1272 & 1 & Flat & Steel & Lattice & WAPA \\
\hline$\$ 170,000$ & $\$ 193,375$ & 230 & 1565 & 1 & Flat & Steel & Lattice & WAPA \\
\hline$\$ 191,000$ & $\$ 217,263$ & 230 & 795 & 2 & Flat & Steel & Lattice & WAPA \\
\hline$\$ 216,000$ & $\$ 245,700$ & 230 & 954 & 2 & Flat & Steel & Lattice & WAPA \\
\hline$\$ 248,000$ & $\$ 282,100$ & 230 & 1272 & 2 & Flat & Steel & Lattice & WAPA \\
\hline$\$ 285,000$ & $\$ 324,188$ & 230 & 1565 & 2 & Flat & Steel & Lattice & WAPA \\
\hline$\$ 192,000$ & $\$ 218,400$ & 345 & 795 & 1 & Flat & Steel & Lattice & WAPA \\
\hline$\$ 209,000$ & $\$ 237.738$ & 345 & 954 & 1 & Flat & Steel & Lattice & WAPA \\
\hline$\$ 231,000$ & $\$ 262,763$ & 345 & 1272 & 1 & Flat & Steel & Lattice & WAPA \\
\hline$\$ 260,000$ & $\$ 295,750$ & 345 & 1555 & 1 & Flat & Steel & Lattice & WAPA \\
\hline$\$ 323,000$ & $\$ 367,413$ & 345 & 795 & 2 & Flat & Steel & Lattice & WAPA \\
\hline$\$ 353,000$ & $\$ 401,538$ & 345 & 954 & 2 & Flat & Steel & Lattice & WAPA \\
\hline$\$ 391.000$ & $\$ 444,763$ & 345 & 1272 & 2 & Flat & Steel & Lattice & WAPA \\
\hline
\end{tabular}


Table A.1. (contd)

\begin{tabular}{|c|c|c|c|c|c|c|c|c|}
\hline RAW Cost & Adjusted Cost & Voltage $(k V)$ & Conductor Size & $\begin{array}{c}\text { Number of } \\
\text { Clrcults }\end{array}$ & $\begin{array}{c}\text { Terrain } \\
\text { Type }\end{array}$ & Pole Type & Frame Type & Agency \\
\hline$\$ 442,000$ & $\$ 502,775$ & 345 & 1565 & 2 & Flat & Steel & Lattice & WAPA \\
\hline$\$ 366,300$ & $\$ 375.738$ & 500 & 3578 & 1 & Flat & Steel & Lattice & BPA \\
\hline$\$ 421,760$ & $\$ 432,627$ & 500 & 5607 & 1 & Flat & Steel & L̦attice & BPA \\
\hline$\$ 431,230$ & $\$ 402,128$ & 500 & 3578 & $i$ & Rolling & Steel & Lattice & BPA \\
\hline$\$ 480,590$ & $\$ 448,157$ & 500 & 5607 & 1 & Rolling & Steel & Lattice & BPA \\
\hline$\$ 449,250$ & $\$ 390,530$ & 500 & 3578 & 1 & $50 / 50^{\circ}$ & Steel & Lattice & BPA \\
\hline$\$ 521,860$ & $\$ 453,649$ & 500 & 5607 & 1 & $50 / 50$ & Steel & Lattice & BPA \\
\hline$\$ 654,860$ & $\$ 569,265$ & 500 & 5607 & 1 & $50 / 50$ & Steel & Single Pole & BPA \\
\hline$\$ 803.450$ & $\$ 824,151$ & 500 & 5607 & 2 & Flat & Steel & Lattice & BPA \\
\hline$\$ 881.680$ & $\$ 904,396$ & 500 & 6039 & 2 & Flat & Steel & Lattice & BPA \\
\hline$\$ 862,530$ & $\$ 884,753$ & 500 & 7218 & 2 & Flat & Steel & Lattice & BPA \\
\hline$\$ 916,170$ & $\$ 854,341$ & 500 & 5607 & 2 & Rolling & Steel & Lattice & BPA \\
\hline$\$ 1,001,500$ & $\$ 933,912$ & 500 & 6039 & 2 & Rolling & Steel & Lattice & BPA \\
\hline$\$ 975,860$ & $\$ 910,003$ & 500 & 7218 & 2 & Rolling & Steel & Lattice & BPA \\
\hline$\$ 961,170$ & $\$ 835,538$ & 500 & 5607 & 2 & $50 / 50^{\circ}$ & Steel & Lattice & BPA \\
\hline$\$ 1,045,840$ & $\$ 909,141$ & 500 & 6039 & 2 & $50 / 50$ & Steel & Lattice & BPA \\
\hline$\$ 1,020,540$ & $\$ 887,148$ & 500 & 7218 & 2 & $50 / 50$ & Steel & Lattice & BPA \\
\hline$\$ 1,061,100$ & $\$ 922,406$ & 500 & 5607 & 2 & $50 / 50$ & Steel & Single Pole & BPA \\
\hline$\$ 290,000$ & $\$ 329,875$ & 500 & 795 & 1 & Flat & Steel & Lattice & WAPA \\
\hline$\$ 306,000$ & $\$ 348,075$ & 500 & 954 & 1 & Flat & Steel & Lattice & WAPA \\
\hline$\$ 327,000$ & $\$ 371,963$ & 500 & 1272 & 1 & Flat & Steel & Lattice & WAPA \\
\hline$\$ 358,000$ & $\$ 407,225$ & 500 & 1565 & 1 & Flat & Stee! & Lattice & WAPA \\
\hline$\$ 389,000$ & $\$ 442,488$ & 500 & 1780 & 1 & Flat & Steel & Lattice & WAPA \\
\hline$\$ 492,000$ & $\$ 559,650$ & 500 & 795 & 2 & Flat & Sleel & Lattice & WAPA \\
\hline$\$ 513,000$ & $\$ 583,538$ & 500 & 954 & 2 & Flat & Steel & Lattice & WAPA \\
\hline$\$ 546,000$ & $\$ 621,075$ & 500 & 1272 & 2 & Flat & Steel & Lattice & WAPA \\
\hline$\$ 599,000$ & $\$ 681,363$ & 500 & 1565 & 2 & Flat & Stee! & Lattice & WAPA \\
\hline$\$ 652,000$ & $\$ 741,650$ & 500 & 1780 & 2 & Flat & Steel & Lattice & WAPA \\
\hline
\end{tabular}


Table A.2. FERC RAW Data

\begin{tabular}{|c|c|c|c|c|c|c|}
\hline Company & Volitage Level (kV) & $\begin{array}{l}\text { Line Length } \\
\text { |(Miles) }\end{array}$ & Land co'st (5000)" & $\begin{array}{c}\text { Structure Cost } \\
(5000)^{\circ}\end{array}$ & $\begin{array}{c}\text { Conductor Cost } \\
\text { (\$000) }\end{array}$ & Total (5000)" \\
\hline Appalachian Power & $\$ 138$ & 6.8 & 315 & 1445 & 772 & 2532 \\
\hline Carolina Power and Light & $\$ 138$ & 15.78 & - & 204 & 153 & 357 \\
\hline Central Illunois Public Service & $\$ 138$ & 34.32 & 1732 & 2986 & 3158 & 7875 \\
\hline Cemtral Power and Light & $\$ 138$ & 59.6 & 151 & 93 & - & 244 \\
\hline CinicinattI Gas and Eloctnc & $\$ 138$ & 3.15 & - & - & - & - \\
\hline Columbus Southem Power & $\$ 138$ & 2.53 & 359 & 275 & 2964 & 3599 \\
\hline Commonweath Edison & $\$ 138$ & 31.85 & - & 371 & 159 & 530 \\
\hline Consolidared Edison Now York & $\$ 138$ & 4.31 & - & 8166 & 6478 & 14644 \\
\hline Consumer Power & $\$ 138$ & 37.1 & 576 & 3273 & 2786 & 6635 \\
\hline Dayon Power and Light & $\$ 138$ & 2.23 & - & $\cdot$ & - & - \\
\hline Del Marva Power and Light & $\$ 138$ & 4.63 & - & - & - $\cdot$ & 2544 \\
\hline Duquesne Light & $\$ 138$ & 7.16 & 149 & 1296 & 1406 & 2851 \\
\hline Flonda Power and Light & $\$ 138$ & 238.88 & 304 & 5419 & 4748 & 10450 \\
\hline Georgla Power & $\$ 138$ & 0.7 & - & - & . & - \\
\hline Hawaiian Electnc & $\$ 138$ & 0.24 & - & - & - & - \\
\hline Houstaon Lighting and Power & $\$ 138$ & - & - & - & - & . \\
\hline Idaho Power & $\$ 138$ & 1.36 & - & $10 t$ & 87 & 188 \\
\hline IIlinois Power & $\$ 138$ & 9.88 & 83 & $3 \times 2$ & 309 & 754 \\
\hline Indaana Michigan Power & $\$ 138$ & 19.58 & 878 & 968 & 1092 & 2938 \\
\hline Indianapolis Power and Light & $\$ 138$ & 10.47 & - & - & - & - \\
\hline Kentucky Power & $\$ 138$ & 1.02 & - & 167 & 240 & 406 \\
\hline Nevada Power & $\$ 138$ & 17.34 & 331 & 1877 & 459 & 2666 \\
\hline Ohio Edison & $\$ 138$ & - & 1. & 97 & 22 & 121 \\
\hline Ohio Power & $\$ 138$ & 6.66 & 443 & 1828 & 1231 & 3502 \\
\hline OKlahoma Gas \& Electric & $\$ 138$ & 4.48 & 1031 & 4320 & 1960 & 7311 \\
\hline Peoples Electric Co-cp & $\$ 138$ & 12.43 & - & - & - & 1540 \\
\hline Public Service Co. of OKlahoma & $\$ 138$ & 10.84 & 347 & 652 & 865 & 1865 \\
\hline Public Service Electric and Gas Co. & $\$ 138$ & - & 75 & 17229 & 6641 & 23945 \\
\hline PSI Energy Inc. & $\$ 138$ & 4 & 66 & 692 & 372 & 1130 \\
\hline Southem Indiana Gas and Electric & $\$ 138$ & 7.08 & - & 591 & 356 & 946 \\
\hline Texas Utilitios Electric & $\$ 138$ & 277.06 & - & - & - & - \\
\hline Uilicorp United Inc. & $\$ 138$ & 02 & 20 & 199 & 108 & 327 \\
\hline Wes Penn Power & $\$ 138$ & 5.3 & 1160 & 2016 & 708 & 3884 \\
\hline West Texas Utiltios & $\$ 138$ & 35.59 & 159 & 1032 & 835 & 2026 \\
\hline Wisconson Electric Power & $\$ 138$ & 11.83 & - & 1677 & 1118 & 2795 \\
\hline Philidabhia Electric & $\$ 220$ & - & 4952 & 8510 & 4692 & 18154 \\
\hline $\begin{array}{l}\text { Alabama Power } \\
\text { Appalachian Power }\end{array}$ & $\$ \$ 2300$ & $\begin{array}{l}5.05 \\
91.42\end{array}$ & 39094 & $\begin{array}{l}1485 \\
9837\end{array}$ & $\begin{array}{l}440 \\
7655\end{array}$ & $\begin{array}{c}1925 \\
21426\end{array}$ \\
\hline $\begin{array}{l}\text { Battimore Gas \& Elocanc } \\
\text { Florida Power and Light }\end{array}$ & $\$ 230$ & $\begin{array}{c}4.5 \\
76.11\end{array}$ & 991 & $\begin{array}{l}3540 \\
2105\end{array}$ & $\begin{array}{l}2789 \\
1600\end{array}$ & $\begin{array}{l}7320 \\
3716\end{array}$ \\
\hline Flonida Power & $\$ 230$ & 034 & - & 93 & 58 & 151 \\
\hline Georgra Power & $\$ 230$ & 7.4 & - & - & 4134 & 4134 \\
\hline Lousiana Power and Light & $\$ 230$ & 31.1 & 23 & 4806 & 3315 & 8145 \\
\hline Mississippi Power and Light & $\$ 230$ & 3.03 & 619 & 683 & 323 & 1626 \\
\hline Montana Power & $\$ 230$ & 4.7 & 580 & 914 & 426 & 1920 \\
\hline Nevada Power & $\$ 230$ & 6.28 & 1025 & 4578 & 2377 & 7980 \\
\hline Norhem States Power & $\$ 230$ & 0.05 & - & 65 & 64 & 129 \\
\hline PGBE & $\$ 230$ & 17.59 & 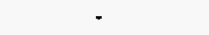 & - & - & - \\
\hline Ponnsylvania Power and Light & $\$ 230$ & 22.23 & - & 293 & 3971 & 4264 \\
\hline Patomic Electric Power & $\$ 230$ & 0.57 & - & 42 & 146 & 188 \\
\hline Public Service Co. of Colorado & $\$ 230$ & 655.06 & 3567 & 31131 & 20258 & 54959 \\
\hline Public Service Eloctrc and Gas Co. & $\$ 230$ & 1041 & 8294 & 10644 & 5127 & 24065 \\
\hline Puget Sound Power and Light & $\$ 230$ & 2281 & 789 & 1373 & 2360 & 4522 \\
\hline PSI Energy Inc. & $\$ 230$ & 3 & - & 1799 & 1199 & 2998 \\
\hline South Carolina Electric and Gas & $\$ 230$ & 8 & 413 & 784 & 1101 & 2278 \\
\hline Virgina Electne and Power & $\$ 230$ & 19.79 & 2194 & 4919 & 3383 & 10495 \\
\hline Boston Edison & $\$ 345$ & 005 & - & & - & $\cdot$ \\
\hline Consolidated Edison New York & $\$ 345$ & - & 36 & 239 & - & 275 \\
\hline Consumers Power & $\$ 345$ & 20 & 247 & 5631 & 2731 & 8609 \\
\hline Ohio Edison & $\$ 345$ & - & - & 303 & 108 & 411 \\
\hline Oklahoma Gas \& Eloctrc & $\$ 345$ & $\cdot$ & 10 & 117 & 2 & 109 \\
\hline Publuc Service Co. of Colorado & $\$ 345$ & 346.7 & 1499 & 21888 & 8291 & 31678 \\
\hline Saint Joseph Lught and Power & $\$ 345$ & 102.99 & $\cdot$ & - & 2594 & 2594 \\
\hline Texas Unlitites Eloctric & $\$ 345$ & 37.68 & - & - & - & - \\
\hline Wisconson Elactric Power & $\$ 345$ & 76.3 & 1629 & 14085 & 15382 & 31096 \\
\hline Puble Sorvice Eloctnc and Gas Co. & $\$ 500$ & - & 22 & & - & 22 \\
\hline
\end{tabular}


Appendix B

Composite Data 


\section{Appendix B}

\section{Composite Data}

This appendix contains the composite data set generated as described in the report. The first table contains the entire data set together; the subsequent tables contain the same data organized by specific design configurations. To arrive at an estimate for total project cost, look up the composite cost in either of these tables for the configuration of interest. Then, refer to the final two pages in this appendix, which contain the additional costs (terrain factor, line length factor, land, environmental, planning, and construction supervision) that must be added to the composite per-mile cost to arrive at a total project cost. For land and environmental costs, both the Bonneville Power Administration (BPA) and the Western Area Power Administration (WAPA) approaches are provided. 
Table B.1. Composite Data Set

\begin{tabular}{|c|c|c|c|c|c|c|c|c|}
\hline $\begin{array}{c}\text { Composite Cost } \\
\text { (Soog) }\end{array}$ & $\begin{array}{c}\text { Voltage } \\
(k V)\end{array}$ & $\begin{array}{c}\text { Conductor } \\
\text { Size }\end{array}$ & $\begin{array}{c}\text { Number of } \\
\text { Circuits }\end{array}$ & $\begin{array}{l}\text { Terrain } \\
\text { Type }\end{array}$ & Pole Type & Frame Type & $\begin{array}{c}\text { Reference } \\
\text { Number/Sheet }\end{array}$ & Source \\
\hline 41 & 13.8 & 123 & 1 & Flat & Wood & Single Pole & 60 & WAPA \\
\hline 42 & 13.8 & 138 & 1 & Flat & Wood & Single Pole & 61 & WAPA \\
\hline 43 & 13.8 & 155 & 1. & Flat & Wood & Single Pole & 62 & WAPA \\
\hline 46 & 13.8 & 174 & 1 & Flat & Wood & Single Pole & 63 & WAPA \\
\hline 48 & 13.8 & 267 & 1 & Flat & Wood & Single Pole & 64 & WAPA \\
\hline 50 & 13.8 & 336 & 1 & Flat & Wood & Single Pole & 65 & WAPA \\
\hline 43 & 34.5 & 123 & 1 & Flat & Wood & Single Pole & 66 & WAPA \\
\hline 46 & 34.5 & 138 & 1 & Flat & Wood & Single Pole & 67 & WAPA \\
\hline 47 & 34.5 & 155 & 1 & Flat & Wood & Single Pole & 68 & WAPA \\
\hline 48 & 34.5 & 174 & 1 & Flat & Wood & Single Pole & 69 & WAPA \\
\hline 50 & 34.5 & 267 & 1 & Flat & Wood & Single Pole & 70 & WAPA \\
\hline 55 & 34.5 & 336 & 1 & Flat & Wood & Single Pole & 71 & WAPA \\
\hline 46 & 46 & 123 & 1 & Flat & Wood & Single Pole & 72 & WAPA \\
\hline 47 & 46 & 138 & 1 & Flat & Wood & Single Pole & 73 & WAPA \\
\hline 48 & 46 & 155 & 1 & Flat & Wood & Single Pole & 74 & WAPA \\
\hline 49 & 46 & 174 & 1 & Flat & Wood & Single Pole & 75 & WAPA \\
\hline 52 & 46 & 267 & 1 & Flat & Wood & Single Pole & 76 & WAPA \\
\hline 56 & 46 & 336 & 1 & Flat & Wood & Single Pole & 77 & WAPA \\
\hline 60 & 69 & 174 & 1 & Flat & Wood & Single Pole & 78 & WAPA \\
\hline 63 & 69 & 267 & 1 & Flat & Wood & Single Pole & 79 & WAPA \\
\hline 66 & 69 & 336 & 1 & Fat & Wood & Single Pole & 80 & WAPA \\
\hline 68 & 69 & 398 & 1 & Flat & Wood & Single Pole & 81 & WAPA \\
\hline 73 & 69 & 477 & 1 & Flat & Wood & Single Pole & 82 & WAPA \\
\hline 75 & 69 & 557 & 1 & Flat & Wood & Single Pole & 83 & WAPA \\
\hline 80 & 69 & 636 & 1 & Flat & Wood & Single Pole & 84 & WAPA \\
\hline 86 & 69 & 795 & 1 & Flat & Wood & Single Pole & 85 & WAPA \\
\hline 68 & 69 & 174 & 1 & Flat & Wood & $\mathrm{H}$-Frame & 91 & WAPA \\
\hline 71 & 69 & 267 & 1 & Flat & Wood & H-Frame & 92 & WAPA \\
\hline 74 & 69 & 336 & 1 & Flat & Wood & H-Frame & 93 & WAPA \\
\hline 76 & 69 & 398 & 1 & Flat & Wood & H-Frame & 94 & WAPA \\
\hline 81 & 69 & 477 & 1 & Fat & Wood & H-Frame & 95 & WAPA \\
\hline 83 & 69 & 557 & 1 & Fat & Wood & H-Frame & 96 & WAPA \\
\hline 88 & 69 & 636 & 1 & Flat & Wood & H-Frame & 97 & WAPA \\
\hline 96 & 69 & 795 & 1 & Flat & Wood & H-Frame & 98 & WAPA \\
\hline 96 & 138 & 398 & 1 & Flat & Wood & H-Frame & 106 & WAPA \\
\hline 100 & 138 & 477 & 1 & Flat & Wood & H-Frame & 107 & WAPA \\
\hline 102 & 138 & 557 & 1 & Flat & Wood & H-Frame & 108 & WAPA \\
\hline 107 & 138 & 636 & 1 & Flat & Wood & H-Frame & 109 & WAPA \\
\hline 114 & 138 & 795 & 1 & Flat & Wood & H-Frame & 110 & WAPA \\
\hline 117 & 138 & 954 & 1 & Flat & Wood & $\mathrm{H}$-Frame & 111 & WAPA \\
\hline 126 & 138 & 1137 & 1 & Flat & Wood & $\mathrm{H}-$ Frame & Special 14 & WAPA \\
\hline 131 & 138 & 1272 & 1 & Fat & Wood & H-Frame & Special 15 & WAPA \\
\hline 141 & 138 & 1509 & 1 & Flat & Wood & H-Frame & Special 16 & WAPA \\
\hline 144 & 138 & 1589 & 1 & Flat & Wood & H-Frame & Special 24 & WAPA \\
\hline 159 & 138 & 1979 & 1 & Flat & Wood & H-Frame & Special 17 & WAPA \\
\hline 176 & 138 & 2406 & 1 & Flat & Wood & H-Frame & Special 18 & WAPA \\
\hline 105 & 161 & 477 & 1 & Fat & Wood & H-Frame & 112 & WAPA \\
\hline 108 & 161 & 557 & 1 & Flat & Wood & H-Frame & 113 & WAPA \\
\hline 111 & 161 & 636 & 1 & Flat & Wood & H-Frame & 114 & WAPA \\
\hline 118 & 161 & 795 & 1 & Flat & Wood & H-Frame & 115 & WAPA \\
\hline 123 & 161 & 954 & 1 & Flat & Wood & H-Frame & 116 & WAPA \\
\hline 131 & 161 & 1137 & 1 & Flat & Wood & H-Frame & Special 19 & WAPA \\
\hline 136 & 161 & 1272 & 1 & Flat & Wood & H-Frame & Special 20 & WAPA \\
\hline 145 & 161 & 1509 & 1 & Flat & Wood & H-Frame & Special 21 & WAPA \\
\hline 148 & 161 & 1589 & 1 & Flat & Wood & H-Frame & Special 25 & WAPA \\
\hline
\end{tabular}


Table B.1. (contd)

\begin{tabular}{|c|c|c|c|c|c|c|c|c|}
\hline $\begin{array}{c}\text { Composite Cost } \\
(\$ 000)\end{array}$ & $\begin{array}{c}\text { Voltage } \\
(k V)\end{array}$ & $\begin{array}{l}\text { Conductor } \\
\text { Size }\end{array}$ & $\begin{array}{l}\text { Number of } \\
\text { Circuits }\end{array}$ & $\begin{array}{l}\text { Terrain } \\
\text { Type }\end{array}$ & Pole Type & Frame Type & $\begin{array}{c}\text { Reference } \\
\text { Number/Sheet }\end{array}$ & Source \\
\hline 163 & 161 & 1979 & 1 & Flat & Wood & H-Frame & Special 22 & WAPA \\
\hline 180 & 161 & 2406 & 1 & Flat & Wood & H-Frame & Special 23 & WAPA \\
\hline 101 & 69 & 398 & 1 & Flat & Steel & Single Pole & 120 & WAPA \\
\hline 106 & 69 & 477 & 1 & Flat & Steol & Single Pole & 121 & WAPA \\
\hline 109 & 69 & 557 & 1 & Flat & Steel & Single Pole & 122 & WAPA \\
\hline 114 & 69 & 636 & 1 & Fat & Steel & Single Pole & 123 & WAPA \\
\hline 119 & $69^{\circ}$ & 795 & 1 & Flat & Steol & Singie Pole & 124 & WAPA \\
\hline 123 & 69 & 954 & 1 & Flat & Steol & Single Pole & 125 & WAPA \\
\hline 126 & 69 & 1114 & 1 & Adjusted & Steel & Single Pole & Special 1 & Composite \\
\hline 129 & 69 & 1272 & 1 & Flat & Steol & Single Pole & 126 & WAPA \\
\hline 126 & 138 & 477 & 1 & Flat & Steel & Single Pole & 133 & WAPA \\
\hline 132 & 138 & 557 & 1 & Fat & Steel & Single Pole & 134 & WAPA \\
\hline 138 & 138 & 636 & 1 & Flat & Steol & Single Pole & 135 & WAPA \\
\hline 148 & 138 & 795 & 1 & Flat & Steel & Single Pole & 136 & WAPA \\
\hline 154 & 138 & 954 & 1 & Fat & Steel & Single Pole & 137 & WAPA \\
\hline 159 & 138 & 1114 & 1 & Flat & Steel & Single Pole & Special 2 & Composite \\
\hline 163 & 138 & 1272 & 1 & Flat & Steel & Single Pole & 138 & WAPA \\
\hline 130 & 161 & 477 & 1 & Flat & Steol & Single Pole & 139 & WAPA \\
\hline 138 & 161 & 557 & 1 & Flat & Steel & Single Pole & 140 & WAPA \\
\hline 149 & 161 & 636 & 1 & Flat & Steel & Single Pole & 141 & WAPA \\
\hline 160 & 161 & 795 & 1 & Fat & Streal & Single Pole & 142 & WAPA \\
\hline 169 & 161 & 954 & 1 & Flat & Stoel & Single Pole & 143 & WAPA \\
\hline 176 & 161 & 1114 & 1 & Adjusted & Stoel & Single Pole & Special 3 & Composite \\
\hline 182 & 161 & 1272 & 1 & Flat & Steel & Single Pole & 144 & WAPA \\
\hline 147 & 69 & 398 & 2 & Flat & Stool & Single Pole & 151 & WAPA \\
\hline 160 & 69 & 477 & 2 & Flat & Steel & Single Pole & $152^{\circ}$ & WAPA \\
\hline 171 & 69 & 557 & 2 & Flat & Steel & Single Pole & 153 & WAPA \\
\hline 187 & 69 & 636 & 2 & Flat & Steel & Single Pole & 154 & WAPA \\
\hline 205 & 69 & 795 & 2 & Flat & Steel & Single Pole & 155 & WAPA \\
\hline 218 & 69 & 954 & 2 & Flat & Steel & Single Pole & 156 & WAPA \\
\hline 217 & 69 & 993 & 2 & Flat & Steel & Single Pole & Special 4 & Composite \\
\hline 230 & 69 & 1114 & 2 & Flat & Steel & Single Pole & Special 5 & Composite \\
\hline 238 & 69 & 1272 & 2 & Flat & Steel & Single Pole & 157 & WAPA \\
\hline 191 & 138 & 477 & 2 & Flat & Steel & Single Pole & 165 & WAPA \\
\hline 210 & 138 & 557 & 2 & Flat & Steel & Single Pole & 166 & WAPA \\
\hline 232 & 138 & 636 & 2 & Flat & Steel & Single Pole & 167 & WAPA \\
\hline 258 & 138 & 795 & 2 & Flat & Steel & Single Pole & 168 & WAPA \\
\hline 281 & 138 & 954 & 2 & Flat & Stoel & Single Pole & 169 & WAPA \\
\hline 278 & 138 & 993 & 2 & Flat & Steel & Single Pole & Special 6 & WAPA \\
\hline 295 & 138 & 1114 & 2 & Flat & Steel & Single Pole & Special 7 & WAPA \\
\hline 308 & 138 & 1272 & 2 & Flat & Steel & Single Pole & 170 & WAPA \\
\hline 207 & 161 & 477 & 2 & Flat & Steel & Single Pole & 171 & WAPA \\
\hline 229 & 161 & 557 & 2 & Flat & Steol & Single Pole & 172 & WAPA \\
\hline 253 & 161 & 636 & 2 & Flat & Steel & Single Pole & 173 & WAPA \\
\hline 281 & 161 & 795 & 2 & Flat & Steel & Single Pole & 174 & WAPA \\
\hline$\cdot 305$ & 161 & 954 & 2 & Flat & Steel & Single Pole & 175 & WAPA \\
\hline 301 & 161 & 993 & 2 & Flat & Steel & Single Pole & Special 8 & WAPA \\
\hline 320 & 161 & 1114 & 2 & Flat & Steel & Single Pole & Special 9 & WAPA \\
\hline 333 & 161 & 1272 & 2 & Flat & Steel & Single Pole & 176 & WAPA \\
\hline 218 & 345 & 795 & 1 & Flat & Steel & - Lattice & 187 & WAPA \\
\hline 238 & 345 & 954 & 1 & Flat & Steel & Lattice & 188 & WAPA \\
\hline 239 & 345 & 993 & 1 & Flat & Steel & Lattice & Special 11 & WAPA \\
\hline 250. & 345 & 1114 & 1 & Flat & Steel & Lattice & Special 12 & WAPA \\
\hline 263 & 345 & 1272 & 1 & Flat & Steel & Lattice & 189 & WAPA \\
\hline 289 & 345 & 1509 & 1 & Flat & Steel & Lattice & Special 13 & WAPA \\
\hline 296 & 345 & 1565 & 1 & Flat & Steel & Lattice & 190 & WAPA \\
\hline 315 & 345 & 1780 & 1 & Flat & Steel & Lattice & Special 10 & WAPA \\
\hline 329 & 345 & 1917 & 1 & Flat & Steel & Lattice & Special 26 & WAPA \\
\hline 376 & 345 & 2406 & 1 & Flat & Steel & Lattice & Special 27 & WAPA \\
\hline
\end{tabular}


Table B.1. (contd)

\begin{tabular}{|c|c|c|c|c|c|c|c|c|}
\hline $\begin{array}{c}\text { Composite Cost } \\
(5000)\end{array}$ & $\begin{array}{c}\text { Voltage } \\
(\mathrm{kV})\end{array}$ & $\begin{array}{l}\text { Conductor } \\
\text { Size }\end{array}$ & $\begin{array}{l}\text { Number' of } \\
\text { Circuits }\end{array}$ & $\begin{array}{l}\text { Terrain } \\
\text { Type }\end{array}$ & Pole Type & Frame Type & $\begin{array}{c}\text { Reference } \\
\text { Number/Sheet }\end{array}$ & Source \\
\hline 367 & 345 & 795 & 2 & Flat & Stoel & Lattice & 200 & WAPA \\
\hline 402 & 345 & 954 & 2 & Flat & Stoel & Lattice & 201 & WAPA \\
\hline 445 & 345 & 1272 & 2 & Flat & Steel & Lattice & 202 & WAPA \\
\hline 503 & 345 & 1565 & 2 & Flat & Steel & Lattice & 203 & WAPA \\
\hline 167 & 115 & 795 & 1 & $50 / 50$ & Steel & Lattice & 12 & BPA \\
\hline 228 & 115 & 795 & 2 & $50 / 50$ & Steel & Lattice & 13 & BPA \\
\hline 220 & 230 & 1589 & 2 & Rolling & Wood & H-Frame & 30 & BPA \\
\hline 569 & 500 & 5607 & 1 & $50 / 50$ & Steel & Single Pole & 49 & BPA \\
\hline 1061 & 500 & 5607 & 2 & $50 / 50$ & Strel & Single Pole & 59 & BPA \\
\hline 91 & 115 & 398 & 1 & Adjusted & Wood & Single Pole & A & Composite \\
\hline 94 & 115 & 477 & 1 & Adjusted & Wood & Single Pole & A & Composite \\
\hline 98 & 115 & 557 & 1 & Adjusted & Wood & Single Pole & A & Composite \\
\hline 101 & 115 & 636 & 1 & Adjusted & Wood & Single Pole & A & Composite \\
\hline 108 & 115 & 795 & 1 & Adjusted & Wood & Single Polè & A & Composite \\
\hline 114 & 115 & 954 & 1 & Adjusted & Wood & Single Pole & A & Composite \\
\hline 122 & 115 & 1137 & 1 & Adjusted & Wood & Single Pole & $A$ & Composite \\
\hline 141 & 115 & 1589 & 1 & Adjusted & Wood & Single Pole & A & Composite \\
\hline 89 & 115 & 336 & 1 & Adjusted & Wood & H-Frame & B & Composite \\
\hline 92 & 115 & 398 & 1 & Adjusted & Wood & H-Frame & B & Composite \\
\hline 95 & 115 & 477 & 1 & Adjusted & Wood & H.Frame & B & Composite \\
\hline 98 & 115 & 557 & 1 & Adjusted & Wood & H-Frame & B & Composite \\
\hline 101 & 115 & 636 & 1 & Adjusted & Wood & H-Frame & B & Composite \\
\hline 107 & 115 & 795 & 1 & Adjusted & Wood & H-Frame & B & Composite \\
\hline 113 & 115 & 954 & 1 & Adjusted & Wood & H-Frame & B & Composite \\
\hline 120 & 115 & 1137 & 1 & Adjusted & Wood & H-Frame & B & Composite \\
\hline 126 & 115 & 1272 & 1 & Adjusted & Wood & H-Frame & $B$ & Composite \\
\hline 135 & 115 & 1509 & 1 & Adjusted & Wood & H-Frame & B & Composite \\
\hline 138 & 115 & 1589 & 1 & Adjusted & Wood & H-Frame & $B$ & Composite \\
\hline 153 & 115 & 1979 & 1 & Adjusted & Wood & H-Frame & B & Composite \\
\hline 170 & 115 & 2406 & 1 & Adjusted & Wood & H-Frame & B & Composite \\
\hline 134 & 115 & 795 & 1 & Adjusted & Steel & Single Pole & C & Composite \\
\hline 124 & 115 & 477 & 1 & Adjusted & Steel & Single Pole & c & Composite \\
\hline 127 & 115 & 557 & 1 & Adjusted & Strel & Single Pole & c & Composite \\
\hline 129 & 115 & 636 & 1 & Adjusted & Steol & Single Pole & C & Composite \\
\hline 139 & 115 & 954 & 1 & Adjusted & Stoel & Single Pole & C & Composite \\
\hline 144 & 115 & 1114 & 1 & Adjusted & Steol & Single Pole & C & Composite \\
\hline 148 & 115 & 1272 & 1 & Adjusted & Stool & Single Pole & C & Composite \\
\hline 129 & 230 & 795 & 1 & Adjusted & Wood & H-Frame & $E$ & Composite \\
\hline 133 & 230 & 954 & 1 & Adjusted & Wood & H-Frame & E & Composite \\
\hline 142 & 230 & 1272 & 1 & Adjusted & Wood & H-Frame & $E$ & Composite \\
\hline 139 & 230 & 1137 & 1 & Adjusted & Wood & H-Frame & $E$ & Composite \\
\hline 149 & 230 & 1509 & 1 & Adjusted & Wood & H-Frame & $E$ & Composite \\
\hline 151 & 230 & 1589 & 1 & Adjusted & Wood & H-Frame & $E$ & Composite \\
\hline 162 & 230 & 1979 & 1 & Adjusted & Wood & H-Frame & $E$ & Composite \\
\hline 174 & 230 & 2406 & 1 & Adjusted & Wood & H-Frame & $E$ & Composite \\
\hline 154 & 230 & 795 & 1 & Adjusted & 'Steel & Lattice & F\&G & Composite \\
\hline 164 & 230 & 954 & 1 & Adjusted & Steel & Lattice & F\&G & Composite \\
\hline 183 & 230 & 1272 & 1 & Adjusted & Steel & Lattice & F\&G & Composite \\
\hline 201 & 230 & 1565 & 1 & Adjusted & Steel & Lattice & F\&G & Composite \\
\hline 166 & 230 & 993 & 1 & Adjusted & Steel & Lattice & F\&G & Composite \\
\hline 174 & 230 & 1114 & 1 & Adjusted & Steel & Lattice & F\&G & Composite \\
\hline 198 & 230 & 1509 & 1 & Adjusted & Steel & Lattice & F\&G & Composite \\
\hline 214 & 230 & 1780 & 1 & Adjusted & Steel & Lattice & $\mathbf{F} \& \mathbf{G}$ & Composite \\
\hline 222 & 230 & 1917 & 1 & Adjusted & Steel & Lattice & F\&G & Composite \\
\hline 252 & 230 & 2406 & 1 & Adjusted & Steel & Lattice & $\mathbf{F} \& \mathbf{G}$ & Composite \\
\hline 254 & 230 & 477 & 1 & Adjusted & Steel & Single Pole & $H$ & Composite \\
\hline 261 & 230 & 557 & 1 & Adjusted & Strel & Single Pole & $\mathrm{H}$ & Composite \\
\hline 268 & 230 & 636 & 1 & Adjusted & Steel & Single Pole & $H$ & Composite \\
\hline 282 & 230 & 795 & 1 & Adjusted & Steel & Single Pole & $\mathrm{H}$ & Composite \\
\hline
\end{tabular}


Table B.1. (contd)

\begin{tabular}{|c|c|c|c|c|c|c|c|c|}
\hline $\begin{array}{c}\text { Composite Cost } \\
(\$ 000)\end{array}$ & $\begin{array}{c}\text { Voltage } \\
(\mathrm{kV})\end{array}$ & $\begin{array}{l}\text { Conductor } \\
\text { Size }\end{array}$ & $\begin{array}{l}\text { Number of } \\
\text { Circuits }\end{array}$ & $\begin{array}{c}\text { Terrain } \\
\text { Type }\end{array}$ & Pole Type & Frame Type & $\begin{array}{c}\text { Reference } \\
\text { Number/Sheat }\end{array}$ & Source \\
\hline 296 & 230 & 954 & 1 & Adjusted & Stoel & Single Pole & H & Composite \\
\hline 324 & 230 & 1272 & 1 & Adjusted & Stoel & Single Pole & $\mathrm{H}$ & Composite \\
\hline 310 & 230 & 1114 & 1 & Adjusted & Steol & Single Pole & H & Composite \\
\hline 352 & 230 & 1589 & 1 & Adjusted & Stoel & Single Pole & H & Composite \\
\hline 254 & 230 & 795 & 2 & Adjusted & Stoel & Single Pole & I\&J & Composite \\
\hline 265 & 230 & 954 & 2 & Adjusted & Steol & Single Pole & I\&J & Composite \\
\hline 289 & 230 & 1272 & 2 & Adjusted & Stgol & Single Pole & I\&J & Composite \\
\hline 310 & 230 & 1565 & 2 & Adjusted & Stzel & Single Pole & I\&J & Composite \\
\hline 268 & 230 & 993 & 2 & Adjusted & Steol & Single Pole & |\&J & Composite \\
\hline 277 & 230 & 1114 & 2 & Adjusted & Stool & Single Pole & I\&J & Composite \\
\hline 306 & 230 & 1509 & 2 & Adjusted & Stoel & Single Pole & I\&J & Composite \\
\hline 336 & 230 & 1917 & 2 & Adjusted & Strol & Single Pole & I\&J & Composite \\
\hline 371 & 230 & 2406 & 2 & Adjusted & Steol & Single Pole & I\&J & Composite \\
\hline 364 & 500 & 795 & 1 & Adjusted & Steol & Lattice & KLM & Composite \\
\hline 367 & 500 & 954 & 1 & Adjusted & Stoel & Lattice & KLM & Composite \\
\hline 367 & 500 & 993 & 1 & Adjustod & Steel & Lattice & KLM & Composite \\
\hline 369 & 500 & 1114 & 1 & Adjusted & Steel & Lattice & KLM & Composite \\
\hline 372 & 500 & 1272 & 1 & Adjusted & Steel & Lattice & $\mathrm{KLM}$ & Composite \\
\hline 376 & 500 & 1509 & 1 & Adjusted & Steel & Lattice & KLM & Composite \\
\hline 376 & 500 & 1565 & 1 & Adjusted & Stoel & Lattice & KLM & Composite \\
\hline 380 & 500 & 1780 & 1 & Adjusted & Strel & Lattice & KLM & Composite \\
\hline 382 & 500 & 1917 & 1 & Adjusted & Stoel & Lattice & KLM & Composite \\
\hline 390 & 500 & 2406 & 1 & Adjusted & Stoel & Lattice & KLM & Composite \\
\hline 408 & 500 & 3578 & 1 & Adjusted & Steel & Lattice & KLM & Composite \\
\hline 440 & 500 & 5607 & 1 & Adjusted & Stool & Lattice & KLM & Composite \\
\hline 370 & 500 & 795 & 2 & Adjusted & Stoel & Lattice & NOP & Composite \\
\hline 385 & 500 & 954 & 2 & Adjusted & Steel & Lattice & NOP & Composite \\
\hline 414 & 500 & 1272 & 2 & Adjusted & Stool & Lattice & NOP & Composite \\
\hline 442 & 500 & 1565 & 2 & Adjusted & Steel & Lattice & NOP & Composite \\
\hline 462 & 500 & 1780 & 2 & Adjustod & Stoel & Lattice & NOP & Composite \\
\hline 821 & 500 & 5607 & 2 & Adjusted & Steel & Lattice & NOP & Composite \\
\hline 861 & 500 & 6039 & 2 & Adjusted & Steel & Lattice & NOP & Composite \\
\hline 972 & 500 & 7218 & 2 & Adjusted & Steel & Lattice & NOP & Composite \\
\hline
\end{tabular}


Table B.2. Composite Cost for Wood Single Pole, Single-Circuit Transmission Lines

\begin{tabular}{|l|l|}
\hline Pole Type & Steel \\
\hline Frame Type & Lattice \\
\hline Number of Circuits & 1 \\
\hline
\end{tabular}

\begin{tabular}{|c|c|c|c|c|}
\hline Composite Cost (\$000) & Voltage $(\mathrm{kV})$ & & & \\
\hline Conductor Size & 175 & 230 & 345 & 500 \\
\hline 95 & 68 & (6) & 48 & 86 \\
\hline Ut & $k x$ & $=9.6$ & $2 \times 3$ & (6) \\
\hline 306 & 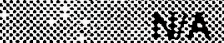 & 1.65 & 209 & 36. \\
\hline (k) & 阷 & 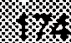 & $(25$ & 60 \\
\hline 878 & $x$ & 19 & $2 x$ & \% \\
\hline$(5,4)$ & 46 & 86 & 289 & 26 \\
\hline 565 & w & 20 & 4 & 6 \\
\hline 8 & 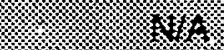 & 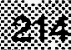 & 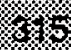 & 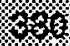 \\
\hline $96 \%$ & * & 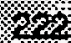 & 4 & $8 \%$ \\
\hline 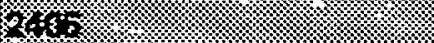 & W & 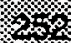 & 秋 & 60 \\
\hline 588 & 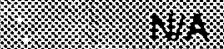 & 好 & 恶 & 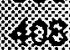 \\
\hline 563 & *2: & 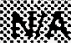 & $x_{x}$ & \% \\
\hline
\end{tabular}

Table B.3. Composite Cost for Wood H-Frame, Single-Circuit Transmission Lines

\begin{tabular}{|l|l|}
\hline Pole Type & Wood \\
\hline Frame Type & H-Frame \\
\hline Number of Circuits & 1 \\
\hline
\end{tabular}

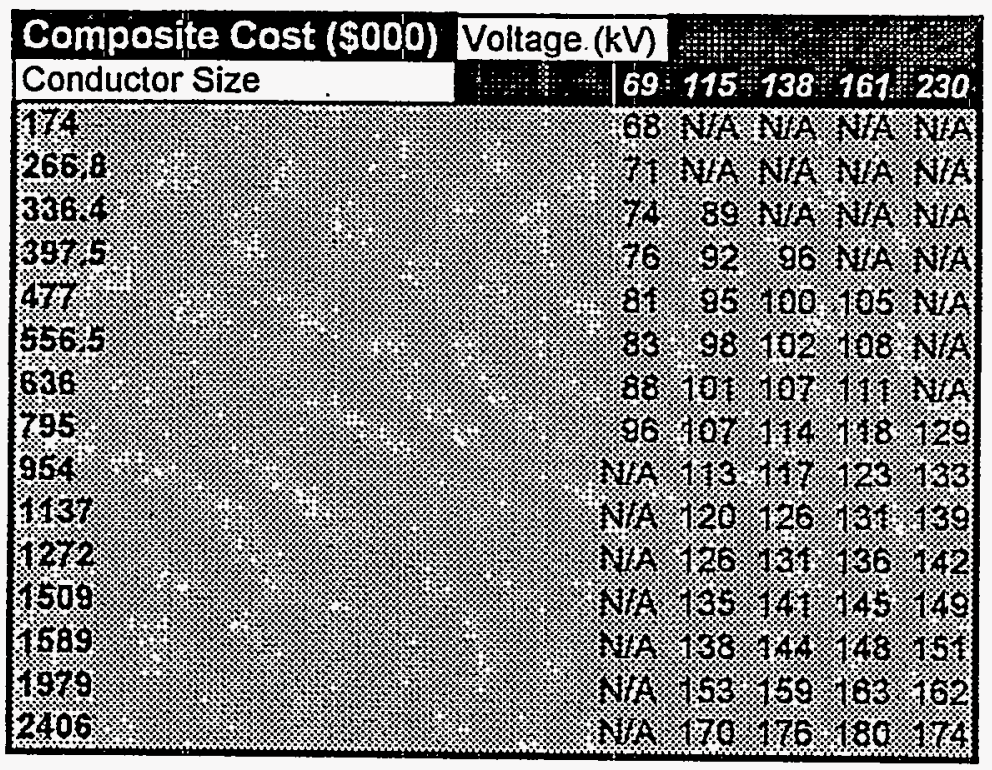

B. 6 
Table B.4. Composite Cost for Wood H-Frame, Single-Circuit Transmission Lines

\begin{tabular}{|l|l|}
\hline Pole Type & Wood \\
\hline Frame Type & H-Frame \\
\hline Number of Circuits & 2 \\
\hline
\end{tabular}

\begin{tabular}{|l|r|}
\hline Composite Cost $(\$ 000)$ & Voltage (kV) \\
\hline Conductor Size & 230 \\
\hline 6589 & \\
\hline
\end{tabular}

Table B.5. Composite Cost for Steel Single Pole, Single-Circuit Transmission Lines

\begin{tabular}{|l|l|}
\hline Pole Type & Steel \\
\hline Frame Type & Single Pole \\
\hline Number of Circuits & 1 \\
\hline
\end{tabular}

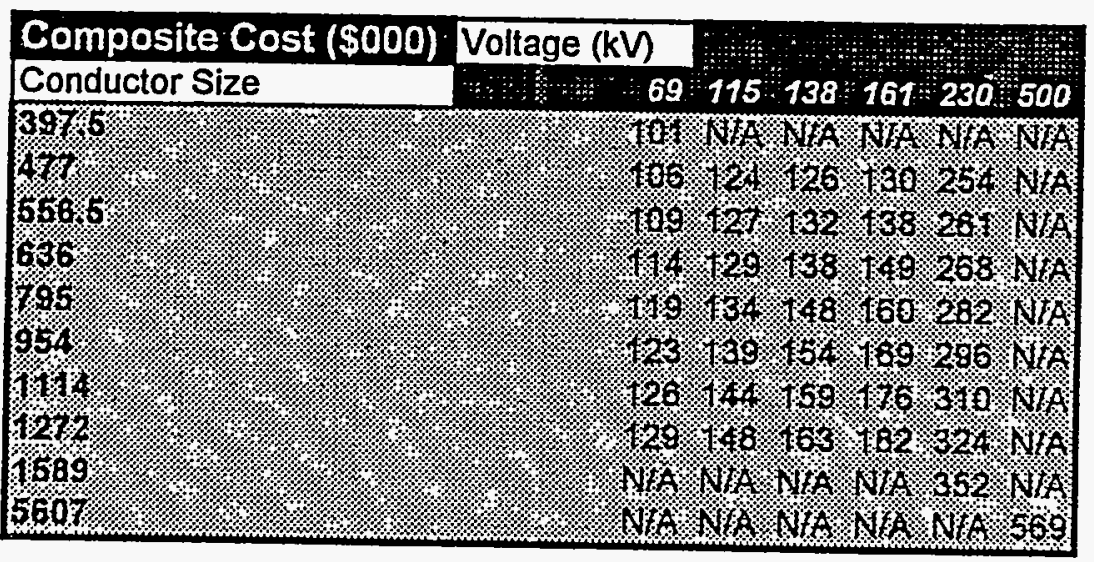

B. 7 
Table B.6. Composite Cost for Steel Single Pole, Double-Circuit Transmission Lines

\begin{tabular}{|l|l|}
\hline Pole Type & Steel \\
\hline Frame Type & Single Pole \\
\hline Number of Circuits & 2 \\
\hline
\end{tabular}

\begin{tabular}{|c|c|c|c|c|c|}
\hline Composite Cost (\$00) & oltage (kV) & & & & \\
\hline Conductor Size & 69 & 138: & 161 & 230 & 500 \\
\hline 96 & 称勧 & Th & 将 & 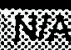 & 10 \\
\hline 472 & 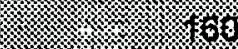 & (1) & 23 & $\sqrt[x]{x}$ & 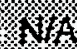 \\
\hline $556 \%$ & 济 & 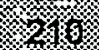 & 829 & 絧 & (x) \\
\hline 636 & 8 & 2.32. & 2153 & 4 & (x) \\
\hline 105 & 28 & 258 & 28 & 25 & 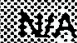 \\
\hline 95 & 到 & 328 ; & 305 & 265 & 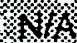 \\
\hline 998 & 2. & $28 \times 3$ & 30 & 268 & 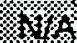 \\
\hline 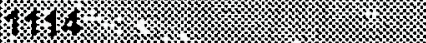 & 230 & 12.95 & 30 & $23 \%$ & 滋滋 \\
\hline 62 & 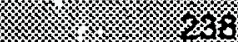 & (3.8 & 的经 & 88 & $x$ \\
\hline 4509 & M & (x) & 准致 & 8393 & 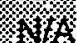 \\
\hline 56 & $1 \times$ & 14 & 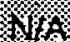 & 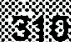 & 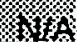 \\
\hline $19+1$ & 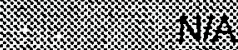 & x & 将 & 36 & (1) \\
\hline 2406 & 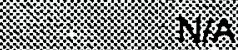 & Nat & 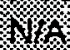 & 6 & 椾 \\
\hline 5607 & 14 & 1 & $y=$ & x & 3 \\
\hline
\end{tabular}

Table B.7. Composite Cost for Steel Lattice, Double-Circuit Transmission Lines

\begin{tabular}{|l|l|}
\hline Pole Type & Steel \\
\hline Frame Type & Lattice \\
\hline Number of Circuits & 1 \\
\hline
\end{tabular}

\begin{tabular}{|c|c|}
\hline Complosite Cost (\$000) & Voltage (kV) \\
\hline Conductor Size & (115 23013451500 \\
\hline 795 & 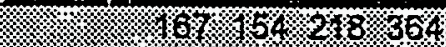 \\
\hline $95 \%$ & 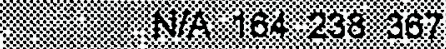 \\
\hline 998 & W \\
\hline $6 / 4 \%$ & $814=1 \%=250 \% 169$ \\
\hline $12 \%$ & 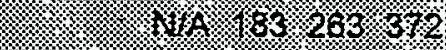 \\
\hline \%og & 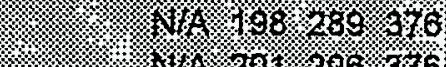 \\
\hline$\frac{1565}{1780}$ & 酒 \\
\hline 1980 & $14=222=329.382$ \\
\hline 2406 & $414=252=366=390$ \\
\hline 3576 & 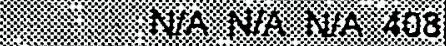 \\
\hline 5607 & 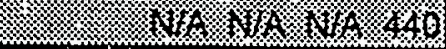 \\
\hline
\end{tabular}

B. 8 
Table B.8. Composite Cost for Steel Lattice, Double-Circuit Transmission Lines

\begin{tabular}{|l|l|}
\hline Pole Type & Steel \\
\hline Frame Type & Lattice \\
\hline Number of Circuits & 2 \\
\hline
\end{tabular}

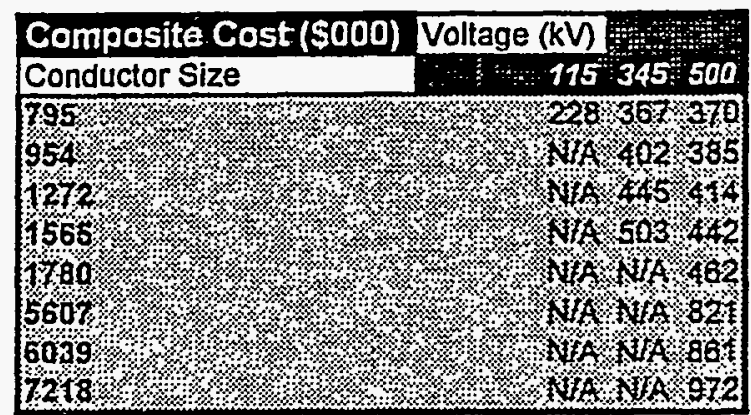

Table B.9. Additional Costs to Add to Per-Mile Principal Contracts Costs from Composite Data Set Terrain Cost Adjustment Multiplier:

Flat Rolfing 5usa Mountainous

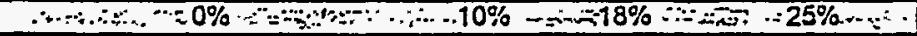

\section{Additional Cost Multipliers:}

Cast Low High

Planning:Construction Supervisión

Note: Field Data, Design and Specifications, and Other Contracts cost multipliers were already added into the composite data set.

\section{Land and Environmental Costs - WAPA Approach} Cost Lou Hiof

Environmental $=0.0 \%$ Land and Rights $=-0.0 \%=0.0 \%=$

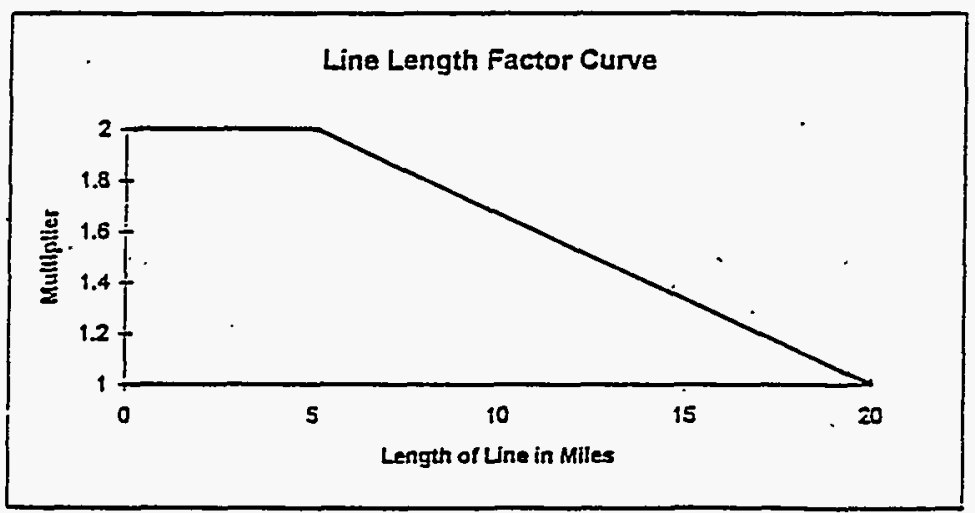


Table B.10. Land Environmental Costs - BPA Approach

\section{Environmental Costs}

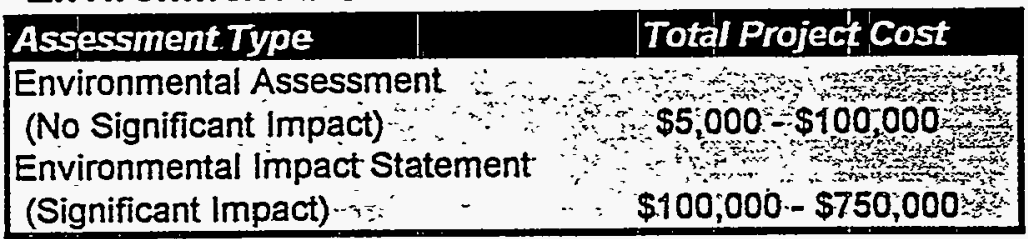

\section{Land Costs}

\begin{tabular}{|c|c|c|}
\hline & $\begin{array}{l}\text { Non-Urban Areas } \\
\text { East of Caspades }\end{array}$ & $\begin{array}{l}\text { Urban Areas } \\
\text { (All West of Cascades) }\end{array}$ \\
\hline $\begin{array}{c}\text { Right-of- } \\
\text { Way Width (ft) }\end{array}$ & $\begin{array}{l}\text { Gregon Washington } \\
(\$ 000) \\
(\$ 000)\end{array}$ & $\begin{array}{cc}\text { Oregon } & \text { Washington } \\
(\$ 000) & (\$ 000)\end{array}$ \\
\hline 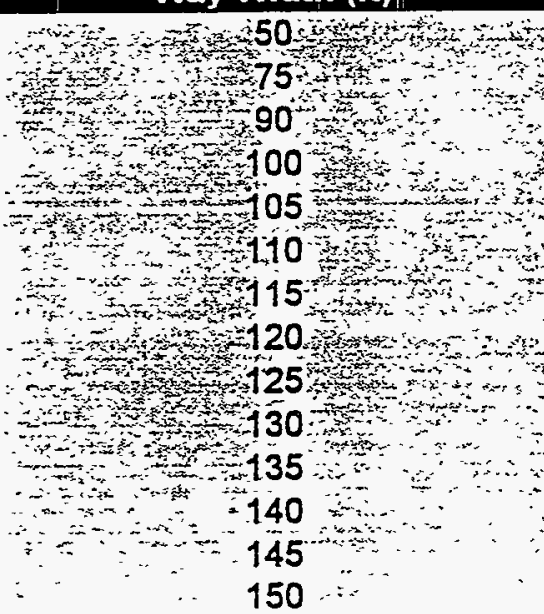 & 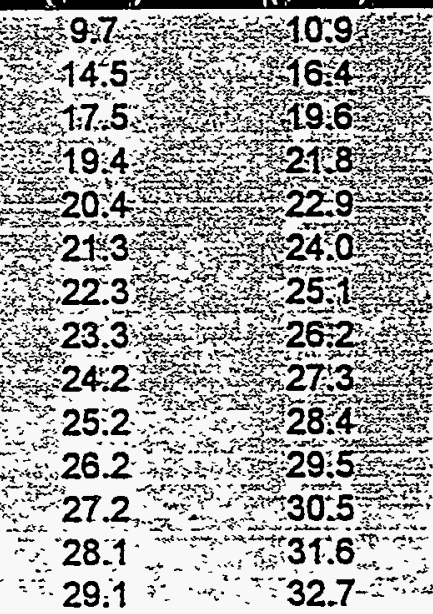 & 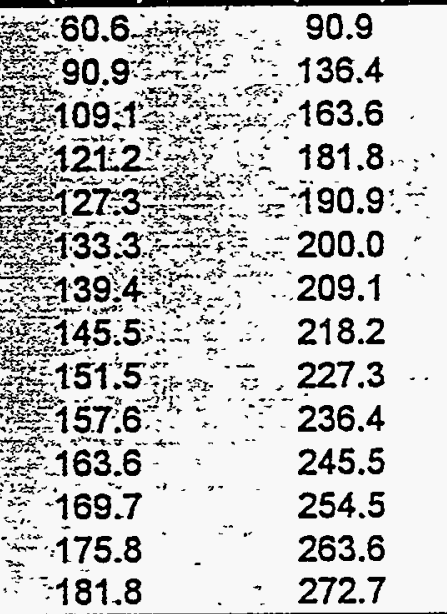 \\
\hline
\end{tabular}

B. 10 
Appendix C

Adjusted FERC Data 


\section{Appendix C}

\section{Adjusted FERC Data}

This appendix contains adjusted Federal Energy Regulatory Commission (FERC) data for comparison with the range of costs estimated by the composite (BPA and WAPA) database. The raw FERC data in Appendix A were updated to 1994 dollars using the Gross Domestic Product (GDP) implicit price deflator and adjusted by the appropriate line length multiplier specified in Figure 2 . 
Table C.1. Adjusted FERC Data

\begin{tabular}{|c|c|c|c|c|}
\hline Company & $\begin{array}{c}\text { Voltage Level } \\
\left.\right|_{i}(\mathbf{k V})\end{array}$ & $\begin{array}{l}\text { Line Length } \\
\text { (Miles) }\end{array}$ & $\begin{array}{c}\text { Compesite Cost of Structure and } \\
\text { Canductor per Mile } \\
\text { (SO0O) }\end{array}$ & $\begin{array}{l}\text { Structure and Conductor Cost Range } \\
\text { from WAPA and BPA for Same Voltage } \\
\text { S(000) }\end{array}$ \\
\hline Appalachian Power & 138 & 6.8 & 182 & $96-308$ \\
\hline Carolina Fowror and Light & 138 & 15.78 & 19 & $96-308$ \\
\hline Central Illinois Public Service & 138 & 34.32 & 188 & $96-308$ \\
\hline Central Power and Light & 138 & 59.6 & - & $96-308$ \\
\hline Cincinarti Gas and Electric & 138 & 3.15 & - & $96-308$ \\
\hline Columbus Southem Power & 138 & 2.53 & 674 & $96-308$ \\
\hline Commonwraith Edison & 138 & 31.85 & 17 & $96-308$ \\
\hline Consolidated Edison New York & 138 & 4.31 & 1787 & $96-308$ \\
\hline Consumers Power & 138 & 37.1 & 172 & $96-308$ \\
\hline Dayton Power and Light & 138 & 2.23 & - & $96-308$ \\
\hline Del Marva Power and Light & 138 & 4.63 & - & 96-308 \\
\hline Duquesno Light & 138 & 7.16 & 214 & $96-308$ \\
\hline Florida Power and Light & 138 & 238.88 & 45 & $96-308$ \\
\hline Georgia Power & 138 & 0.7 & - & $96-308$ \\
\hline Hawailan Eectric & 138 & 0.24 & - & $96-308$ \\
\hline Houston Lightung and Power & 138 & - & - & $96-308$ \\
\hline Idaho Powror & 138 & 1.36 & 73 & $96-308$ \\
\hline Illinois Power & 138 & 9.88 & 43 & 96-308 \\
\hline Inciana Michigan Power & 138 & 19.58 & 108 & $96-308$ \\
\hline Indianapolis Power and Light & 138 & 10.47 & $\cdot$ & $96-308$ \\
\hline Kentucky Power & 138 & 1.02 & 209 & $96-308$ \\
\hline Nevada Powrer & 138 & 17.34 & 120 & $96-308$ \\
\hline Ohio Edison & 138 & - & $\cdot$ & $96-308$ \\
\hline Ohio Pówer & 138 & 6.66 & 256 & $96-308$ \\
\hline Okahoma Gas \& Eloctric & 138 & 4.48 & 737 & $96-308$ \\
\hline Peoples Eectic Co-op & 138 & 12.43 & $\cdot$ & $96-308$ \\
\hline Public Servico Co. of ONahoma & 138 & 10.84 & 91 & $96-308$ \\
\hline Public Servico Electric and Gas Co. & 138 & - & $\cdot$ & $96-308$ \\
\hline PSI Enorgy lne. & 138 & 4 & 140 & $96 \cdot 308$ \\
\hline Southem Indiana Gas and Eloctric & 138 & 7.08 & 76 & $96-308$ \\
\hline Texas Utitities Electric & 138 & 277.06 & $\cdot$ & $96-308$ \\
\hline Utilicorp United lne. & 138 & 0.2 & 806 & $96-308$ \\
\hline Wes Penn Power & 138 & 5.3 & 273 & $96-308$ \\
\hline West Toxas Utilitios & 138 & 35.59 & 55 & $96-308$ \\
\hline Wisconson Electrie Power & 138 & 11.83 & 161 & $96-308$ \\
\hline Philadolohua Electric & 220 & - & $\dot{-}$ & $129-371$ \\
\hline Alabama Power & 230 & 5.05 & 201 & 129-371 \\
\hline Appalachian Powrer & 230 & 91.42 & 201 & $129-371$ \\
\hline Baltumore Gas \& Electric & 230 & 4.5 & 740 & $129-371$ \\
\hline Fonda Powor and Light & 230 & 76.11 & si & $129-371$ \\
\hline Flonda Power & 230 & 0.34 & 234 & $129-371$ \\
\hline Goorgia Power & 230 & 7.4 & - & $129-371$ \\
\hline Louisiana Power and Light & 230 & 31.1 & 275 & $129-371$ \\
\hline Mississippi Power and Light & 230 & 3.03 & 175 & $129-371$ \\
\hline Montana Power & 230 & 4.7 & 150 & $129-371$ \\
\hline Nevada Power & 230 & 6.28 & 609 & $129-371$ \\
\hline Northem States Power & 230 & 0.05 & 1356 & $129-371$ \\
\hline PG\&E & 230 & 17.59 & - & $129-371$ \\
\hline Pennsytvania Power and Light & 230 & 22.23 & 202 & $129-371$ \\
\hline Patomic Electric Power & 230 & 0.57 & 173 & $129-371$ \\
\hline Public Service Co. of Colorado & 230 & 655.06 & 83 & $129-371$ \\
\hline Public Service Electne and Gas Co. & 230 & $10.4 t$ & 972 & $129-371$ \\
\hline Puget Sound Power and Light & 230 & 22.81 & 172 & $129-371$ \\
\hline PSI Energy Inc & 230 & 3 & 526 & $129-371$ \\
\hline South Carolina Electric and Gas & 230 & 8 & 138 & $129-371$ \\
\hline Virgnia Eloctric and Powor & 230 & 19.79 & 435 & $129-371$ \\
\hline goston Edison & 345 & 0.05 & $\cdot$ & $218-503$ \\
\hline Consolidated Edison New York & 345 & - & - & 218.503 \\
\hline Consumers Power & 345 & 20 & 440 & 218.503 \\
\hline Ohio Edison & 345 & - & - & $218-503$ \\
\hline Okahoma Gas \& Eloctric & 345 & $\cdot$ & - & $218-503$ \\
\hline Public Service Co. of Colorado & 345 & 346.7 & 92 & 218-503 \\
\hline Saint Joseph Light and Power & 345 & 102.99 & $\cdot$ & 218.503 \\
\hline Toxas Uitities Eloctne & 345 & 37.68 & - & $218-503$ \\
\hline Wisconson Eactnc Powrer & 345 & 76.3 & 406 & $218-503$ \\
\hline Public Sermes Electnc and Gas Co. & 500 & - & - & $364-972$ \\
\hline
\end{tabular}




\section{Distribution}

No. of

Copies

\section{OFFSITE}

12 DOE/Office of Scientific and Technical Information

A. Akhil

Senior Member of Technical Staff

Division 2525

Sandia National Laboratories

Albuquerque, NM 87185

J. Badin

Program Director

Energetics, Inc.

7164 Columbia Gateway Drive

Columbia, MD 21046

P. Booher

Program Analyst

U.S. Department of Energy, EE-14 1000 Independence Avenue, S.W.

Washington, DC 20585

R. Brewer

Director, Utility Systems Division

U.S. Department of Energy, EE-14 1000 Independence Avenue, S.W.

Washington, DC 20585

L. Coles

NREL

1617 Cole Boulevard

Golden, CO 80401

J. Daley

Program Manager, HTS

U.S. Department of Energy, EE-14

1000 Independence Avenue, S.W.

Washington, DC 20585
No. of

Copies

R. Eaton

U.S. Department of Energy, EE-14

Office of Energy Management

1000 Independence Avenue, S.W.

Washington, DC 20585

C. Gregoire

Senior Chemical Engineer

NREL

1617 Cole Boulevard

Golden, CO 80401

R. Hawsey

Mgr., Superconductivity Program

Oak Ridge National Laboratory

MS 6040

P.O. Box 2008

Oak Ridge, TN 37831-6040

K. Humphreys

Program Support Specialist

U.S. Department of Energy, EE-14 1000 Independence Avenue, S.W.

Washington, DC 20585

J. Hurwitch

Assistant Vice President

Energetics, Inc.

7164 Gateway Drive

Columbia, MD 21046

P. Overholt

Program Manager, T\&D

U.S. Department of Energy, EE-14

1000 Independence Avenue, S.W.

Washington, DC 20585 
PNL-10561

UC-900

No. of

Copies

N. Rossmeissl

U.S. Department of Energy, EE-14 1000 Independence Avenue, S.W.

Washington, DC 20585

R. Sen

President

R.K. Sen \& Associates, Inc.

4733 Bethesda Avenue, Suite 608

Bethesda, MD 20814

J. VanCoevering

Manager, Power Systems

Technical Program

Oak Ridge National Laboratory

Building 3147, MS 6070

P.O. Box 2008

Oak Ridge, TN $37831-6070$
No. of

Copies

\section{ONSITE}

DOE Richland Operations Office

J. K. Schmitz K8-50

23 Pacific Northwest Laboratory

D. R. Brown K8-18

M. L. Brown K9-62

J. G. DeSteese K5-20

L. L. Fassbender K8-18

J. F. Hauer K5-02

K. R. Hughes (10) K8-18

C. H. Imhoff K9-62

L. D. Kannberg K5-20

Publishing Coordination

Technical Report Files (5) 